Draft Version OCtOBER 2, 2018

Preprint typeset using IATEX style emulateapj v. 11/26/03

\title{
SURVEY FOR TRANSITING EXTRASOLAR PLANETS IN STELLAR SYSTEMS: III. A LIMIT ON THE FRACTION OF STARS WITH PLANETS IN THE OPEN CLUSTER NGC 1245
}

\author{
Christopher J. Burke ${ }^{1}$, B. Scott Gaudi ${ }^{2,3}$, D. L. DePoy ${ }^{1}$, and Richard W. Pogge \\ Draft version October 2, 2018
}

\begin{abstract}
We analyze a 19-night photometric search for transiting extrasolar planets in the open cluster NGC 1245. An automated transit search algorithm with quantitative selection criteria finds six transit candidates; none are bona fide planetary transits. We characterize the survey detection probability via Monte Carlo injection and recovery of realistic limb-darkened transits. We use this to derive upper limits on the fraction of cluster members with close-in Jupiter-radii, $R_{J}$, companions. The survey sample contains $\sim 870$ cluster members, and we calculate $95 \%$ confidence upper limits on the fraction of these stars with planets by assuming the planets have an even logarithmic distribution in semimajor axis over the Hot Jupiter (HJ $-3.0<P /$ day $<9.0$ ), Very Hot Jupiter (VHJ $-1.0<P /$ day $<3.0$ ), and an as of yet undetected Extremely Hot Jupiter (EHJ - $P_{\text {Roche }}<P<1.0$ day) period ranges. For $1.5 R_{J}$ companions we limit the fraction of cluster members with companions to $<1.5 \%,<6.4 \%$, and $<52 \%$ for EHJ, VHJ, and HJ companions, respectively. For $1.0 R_{J}$ companions, we find $<2.3 \%$ and $<15 \%$ have EHJ and VHJ companions, respectively. We do not reach the sensitivity to place any meaningful constraints on $1.0 R_{J}$ HJ. From a careful analysis of the random and systematic errors of the calculation, we show that the derived upper limits contain a $\pm_{70}^{13 \%}$ relative error. For similar photometric noise and weather properties as this survey, observing NGC 1245 twice as long results in a tighter constraint on HJ companions than observing an additional cluster of similar richness as NGC 1245 for the same length of time as this survey. If $1 \%$ of stars have $1.5 R_{J}$ HJ companions, we expect to detect one planet for every 5000 dwarf stars observed for a month. To reach a $\sim 2 \%$ upper limit on the fraction of stars with $1.5 R_{J}$ companions in the $3.0<P$ /day $<9.0$ range, we conclude a total sample size of $\sim 7400$ dwarf stars observed for at least a month will be needed. Results for 1.0 $R_{J}$ companions, without substantial improvement in the photometric precision, will require a small factor larger sample size.

Subject headings: open clusters and associations:individual (NGC 1245) — planetary systems — methods: data analysis
\end{abstract}

\section{INTRODUCTION}

Extrasolar planet detections and analysis of the nondetections further our knowledge of the planet formation process and contribute to an empirical determination of the typical planetary system. These empirical constraints will eventually decide the ubiquity or rarity of planetary bodies in the Universe. A variety of techniques exist to detect extrasolar planets (Perrvman 2000), and there are currently 168 extrasolar planet discoveries ${ }^{4}$.

Most of the extrasolar planets have been discovered using the radial velocity technique. Radial velocity detections indicate that $1.2 \% \pm 0.3 \%$ of FGK main-sequence stars in the solar neighborhood have a "Hot" Jupitermass planet (HJ) orbiting within 0.1 AU (Marcy et al. 2005). At this small separation from the central star, the high temperatures and low disk column densities prevent in situ formation of HJ planets (Bodenheimer et al. 2000). Several mechanisms exist to exchange angular momentum between the protoplanet and natal disk, enabling the protoplanet to migrate from a more likely formation separation (several $\mathrm{AU}$ ) to within $0.1 \mathrm{AU}$

1 Astronomy Department, The Ohio State University, $140 \mathrm{~W}$. 18th Ave., Columbus, OH 43210

2 Donald H. Menzel Postdoctoral Fellow

3 Harvard-Smithsonian Center for Astrophysics, 60 Garden Street, Cambridge, MA 02138

Electronic address: cjburke@astronomy.ohio-state.edu

${ }^{4}$ http://www.obspm.fr/encycl/catalog.html
(Terquem et al. 2000). Due to tidal circularization, HJ have nearly circular orbits with an observed median eccentricity, $\langle e\rangle=0.07$, whereas planets with larger separations have a median eccentricity of $\langle e\rangle=0.25$.

In addition to the detection statistics and planet properties, the extrasolar planet detections indicate several physical relationships between the stellar host properties and the frequency of extrasolar planets. The most striking of these is that the probability for hosting an extrasolar planet increases rapidly with stellar metal abundance, consistent with $P \propto N_{\mathrm{Fe}}^{2}$ (Fischer \& Valenti 2005). The frequency of planets may also depend on the stellar mass. Butler et al. (2004) and Bonfils et al. (2005) point out that there exists a deficit of $M_{J}$ planets orbiting $\mathrm{M}$ dwarf stars. However, the increasing number of shortperiod Neptune-mass planets being discovered around $\mathrm{M}$ dwarfs suggests that the overall frequency of planets (of all masses) orbiting $\mathrm{M}$ dwarfs may be similar to FGK dwarfs, but the typical planet mass is less, thereby escaping detection given the detection limitations of the current radial velocity surveys (Bonfils et al. 2005). Additionally, none of the $\mathrm{M}$ dwarfs harboring planets are metal rich Bonfils et al. (2005).

A coherent theory of planet formation and survival requires not only reproducing the physical properties of the planets, but reproducing any trends in the physical properties on the host environment. Despite the knowledge and constraints on extrasolar planets that radial velocity 
surveys provide, radial velocity surveys have their limitations. The high resolution spectroscopic requirements of the radial velocity technique limit its use to the solar neighborhood and orbital periods equivalent to the lifetime of the survey. A full consensus of the planetary formation process requires relying on additional techniques to detect extrasolar planets in a larger variety of conditions prevalent in the Universe.

For instance, microlensing surveys are sensitive to extrasolar planets orbiting stars in the Galactic disk and bulge with distances of many $\mathrm{kpc}$ away (Mao \& Pacvzński 1991; Gould \& Loeb 1992). Two objects consistent with Jupiter-mass companions have been detected via the microlensing technique (Bond et al. 2004; Udalski et al. 2005). Additional information is obtained from studying the microlensing events that did not result in extrasolar planet detections. Microlensing surveys limit the fraction of $\mathrm{M}$ dwarfs in the Galactic bulge with Jupiter-mass companions orbiting between 1.5 AU to 4 AU to $<33 \%$ (Albrow et al. 2001; Gaudi et al. 2002).

Although limited to the solar neighborhood, attempts to directly image extrasolar planets are sensitive to planets with semimajor axis beyond 20 AU. The light from the parent star limits detecting planets interior to the seeing disk. Adaptive optics observations of young $(\sim 1$ Myr) stars provide the best opportunity to directly image extrasolar planets since the young planets are still relatively bright while undergoing a rapid, cooling contraction. Although the interpretation relies on theoretical modeling of these complex planetary objects, three sources in nearby star forming regions have been detected whose broad-band colors and spectra are consistent with those expected from 1-42 Jupiter-mass objects (Neuhäuser, et al. 2005; Chauvin et al. 2005a b). The contrast ratios necessary for extrasolar planet detection are difficult to reach, and results for detecting higher mass brown dwarfs are more complete. An analysis of the Cornell High-Order Adaptive Optics Survey (CHAOS) derives a brown dwarf companion upper limit of $10 \%$ orbiting between 25 and $100 \mathrm{AU}$ of the parent star (Carson et al. 2005). McCarthy \& Zuckerman (2004) estimate $1 \% \pm 1 \%$ of $\mathrm{G}, \mathrm{K}$, and $\mathrm{M}$ stars have brown dwarf companions orbiting between 75 and $300 \mathrm{AU}$, but this estimate may not account for the full range of orbital inclination and eccentricities possible (Carson et al. 2005). At greater separations, > 1000 AU, brown dwarf companions to F-M0 main-sequence stars appear to be as common as stellar companions (Gizis et al. 2001).

After the radial velocity technique, the transit technique has had the most success in detecting extrasolar planets (Konacki et al. 2005). The transit technique can detect $R_{J}$ transits in any stellar environment where $\lesssim 1 \%$ photometry is possible. Thus, it provides the possibility of detecting extrasolar planets in the full range of stellar conditions present in the Galaxy: the Solar neighborhood, the thin and thick disk, open clusters, the halo, the bulge, and globular clusters are all potential targets for transit surveys. A major advantage of the transit technique is the current large-format mosaic CCD imagers which provide multiplexed photometric measurements with sufficient accuracy across the entire field of view.

The first extrasolar planet detections via the tran- sit technique began with the candidate list provided by the OGLE collaboration (Udalski et al. 2002). However, confirmation of the transiting extrasolar planet candidates requires radial velocity observations. Due to the well known equation-of-state competition between electron degeneracy and ionic Coulomb pressure, the radius of an object becomes insensitive to mass across the entire range from below $M_{J}$ to the hydrogen-burning limit (Chabrier \& Baraffe 2000). Thus, objects revealing a $R_{J}$ companion via transits may actually have a brown-dwarf mass companion when followed up with radial velocities. This degeneracy is best illustrated by the planet-sized brown dwarf companion to OGLE-TR-122 (Pont et al. 2005). The first radial-velocity confirmations of planets discovered by transits (Konacki et al. 2003; Bouchy et al. 2004) provided a first glimpse at a population of massive, very close-in planets with $P<3$ days and $M_{p}>M_{J}$ ("Very Hot Jupiters" - VHJ) that had not been seen by radial velocity surveys. Gaudi et al. (2005) demonstrated that, after accounting for the strong sensitivity of the transit surveys to the period of the planets, the transit detections were likely consistent with the results from the radial velocity surveys, implying that VHJs were intrinsically very rare. Subsequently, in a metallicitybiased radial velocity survey, Bouchy et al. (2005b) discovered a VHJ with $P=2.2$ day around the bright star HD189733 that also has observable transits.

Despite the dependence of transit detections on radial velocity confirmation, radial velocity detections alone only result in a lower limit on the planetary mass, and thus do not give a complete picture of planet formation. The mass, radius information directly constrains the theoretical models, whereas either parameter alone does little to further constrain the important physical processes that shape the planet properties (Guillot 2005). For example, the mass-radius relation for extrasolar planets can constrain the size of the rocky core present (e.g., Laughlin et al. 2005). Also, the planet transiting across the face of its parent star provides the exciting potential to probe the planetary atmospheric absorption lines against the stellar spectral features (Charbonneau et al. 2002; Deming et al. 2005a; Narita et al. 2005). Or, in the opposite case, emission from the planetary atmosphere can be detected when the planet orbits behind the parent star (Charbonneau et al. 2005: Deming et al. 2005b).

Despite these exciting results, the transit technique is significantly hindered by the restricted geometrical alignment necessary for a transit to occur. As a result, a transit survey necessarily contains at least an order of magnitude more non-detections than detections. In addition, null results themselves can provide important constraints. For example, the null result in the globular cluster 47 Tucanae adds important empirical constraints to the trend of increasing probability of having a planetary companion with increasing metallicity (Gilliland et al. 2000; Santos et al. 2004). Thus, understanding the sensitivity of a given transit survey, i.e. the expected rate of detections and non-detections, takes on increased importance. Several studies have taken steps toward sophisticated Monte Carlo calculations to quantify detection probabilities in a transit survey (Gilliland et al. 2000; Weldrake et al.|2005; Mocheiska et al. 2005; Hidas et al. 2005; Hood et al. 2005). Unfortunately, these studies do not fully characterize the sources of error and systemat- 
ics present in their analysis, and therefore the reliability of their conclusions is unknown. Furthermore, essentially all of the previous studies have either (1) not accurately determined the number of dwarf main-sequence stars in their sample, or (2) made simplifying assumptions which may lead to misestimated detection probabilities, or (3) contained serious conceptual errors in the procedure with which they have determined detection probabilities, or (4) some combination of the above.

As a specific and important example, most studies do not apply identical selection criteria when searching for transits amongst the observed light curves and when recovering injected transits as part of determining the survey sensitivity. Removal of false-positive transit candidates arising from systematic errors in the light curve has typically involved subjective visual inspections, and these subjective criteria have not been applied to the recovery of injected transits when determining the survey sensitivity. This is statistically incorrect, and can in principle lead to overestimating the survey sensitivity. Even if identical selection criteria are applied to the original transit search and in determining the survey sensitivity, some surveys do not apply conservative enough selections to fully eliminate false-positive transit detections.

In this paper, we address these shortcomings of previous studies in our analysis of a 19-night photometric search for transiting extrasolar planets in the open cluster NGC 1245. An automated transit search algorithm with quantitative selection criteria finds six transit candidates; none are bona fide planetary transits. We describe our Monte Carlo calculation to robustly determine the sensitivity of our survey, and use this to derive upper limits on the fraction of cluster members with close-in, Jupiter-radii, $R_{J}$, companions.

Leading up to the process of calculating the upper limit, we develop several new analysis techniques. First, we develop a differential photometry method that automatically selects comparison stars to reduce the systematic errors that can mimic a transit signal. In addition, we formulate quantitative transit selection criteria, which completely eliminate false positives due to systematic light-curve variability without human intervention. We characterize the survey detection probability via Monte Carlo injection and boxcar recovery of transits. Distributing the Monte Carlo calculation to multiple processors enables rapid calculation of the transit detection probability for a large number of stars.

The techniques developed here enable combining results from transit surveys in a statistically meaningful way. This work is part of the Survey for Transiting Extrasolar Planets in Stellar Systems (STEPSS). The project concentrates on stellar clusters since they provide a large sample of stars of homogeneous metallicity, age, and distance (Burke et al. 2003, 2004). Overall, the project's goal is to assess the frequency of close-in extrasolar planets around main-sequence stars in several open clusters. By concentrating on main-sequence stars in open clusters of known (and varied) age, metallicity, and stellar density, we will gain insight into how these various properties affect planet formation, migration, and survival.

The survey characteristics and the photometric procedure are given in 92 We explain the automated algorithm to calculate the differential light curves and de- scribe the light curve noise properties in $\$ 3$ In $\$ 4$ we describe our implementation of the box-fitting least squares (BLS) method (Kovács et al. 2002) for transit detection. In 4.2 we present a thorough discussion of the quantitative selection criteria for transit detection, followed by a discussion of the objects with sources of astrophysical variability that meet the selection criteria in 95 . We outline the Monte Carlo calculation for determining the detection probability of the survey in 6. We present upper limits for a variety of companion radii and orbital periods in 97. A discussion of the random and systematic errors present in the technique is given in 88 . We compare the final results of this study to our expected detection rate before the survey began and discuss the observations necessary to reach sensitivities similar to radial velocity detection rates in 9. Finally, 910 briefly summarizes this work.

\section{OBSERVATIONS AND DATA REDUCTION}

\subsection{Observations}

We observed NGC 1245 for 19 nights between 24 Oct. and 11 Nov. of 2001 using the MDM 8K mosaic imager on the MDM 2.4m Hiltner telescope. The MDM $8 \mathrm{~K}$ imager consists of a 4x2 array of thinned, 2048x4096, SITe ST002A CCDs (Crotts 2001). This instrumental setup yields a $26^{\prime} \times 26^{\prime}$ field of view and $0.36^{\prime \prime}$ per pixel resolution in 2x2 pixel binning mode. Table 1 has an entry for each night of observations that shows the number of exposures obtained in the cousins $I$-band filter, median full-width-at-half-maximum (FWHM) seeing in arcseconds, and a brief comment on the observing conditions. In total, 936 images produced usable photometry with a typical exposure time of $300 \mathrm{~s}$.

\subsection{Data Reduction}

We use the $\operatorname{IRAF}^{5}$ CCDPROC task for all CCD processing. The read noise measured in zero-second images taken consecutively is consistent with read noise measured in zero-second images spread through the entire observing run. Thus, the stability of the zero-second image over the course of the 19 nights allows median combining 95 images to determine a master, zero-second calibration image. For master flat fields, we median combine 66 twilight sky flats taken throughout the observing run. We quantify the errors in the master flat field by examining the night-to-night variability between individual flat fields. The small-scale, pixel-to-pixel variations in the master flat fields are $\sim 1 \%$, and the large-scale, illumination-pattern variations reach the $3 \%$ level. The large illumination-pattern error results from a sensitivity in the illumination pattern to telescope focus. However, such large-scale variations do not affect differential photometry with proper reference-star selection (as described in $\$ 3$.

To obtain raw instrumental photometric measurements, we employ an automated reduction pipeline that uses the DoPHOT PSF fitting package (Schecter et al. 1993). Comparable quality light curves resulted from photometry via the DAOPHOT/ALLFRAME, PSFfitting photometry packages (Stetson 1987; Stetson et al.

\footnotetext{
5 IRAF is distributed by the National Optical Astronomy Observatories, which are operated by the Association of Universities for Research in Astronomy, Inc., under cooperative agreement with the National Science Foundation.
} 
1998) in the background limited regime. DoPhot performs slightly better in terms of rms scatter in the differential light curve in the source-noise limited regime. The photometric pipeline originated from a need to produce real-time photometry of microlensing events in order to search for anomalies indicating the presence of an extrasolar planet around the lens (Albrow et al. 1998). This study uses a variant of the original pipeline developed at The Ohio State University and currently in use by the Microlensing Follow Up Network (Yoo et al. 2004).

In brief, the pipeline takes as input a high signal-tonoise (S/N) "template" image. A first pass through DoPHOT identifies the brightest, non-saturated stars on all the images. Using these bright-star lists, an automated routine (J. Menzies, private communication) determines the geometric transformation between the template image and all the other other images. A second, deeper pass with DoPhot on the template image identifies all the stars on the template image for photometric measurement. The photometric procedure consists of transforming the deep-pass star list from the template image to each frame. These transformed positions do not vary during the photometric solution. Next, an automated routine (J. Menzies, private communication) determines an approximate value for the FWHM and sky as required by DoPHOT. Finally, DoPHOT iteratively determines a best-fit, 7-parameter analytic PSF and uses this best-fit PSF to determine whether an object is consistent with a single star, double star, galaxy, or artifact in addition to the photometric measurement of the object.

\section{DIFFERENTIAL PHOTOMETRY}

In its simplest form, differential photometry involves the use of a single comparison star in order to remove the time variable atmospheric extinction signal from the raw photometric measurements (Kieldsen \& Frandsen 1992). The process of selecting comparison stars typically consists of identifying an ensemble of bright, isolated stars that demonstrate long term stability over the course of the observations (Gilliland \& Brown 1988). This procedure is sufficient for studying many variable astrophysical sources where several percent accuracy is typically adequate. However, after applying this procedure on a subset of the data, systematic residuals remained in the data that were similar enough in shape, time scale, and depth to the expected signal from a transiting companion to result in large number of highly-significant false positive detections.

Removing $\lesssim 0.01 \mathrm{mag}$ systematic errors resembling a transit signal requires a time consuming and iterative procedure for selecting the comparison ensemble. Additionally, a comparison ensemble that successfully eliminates systematic errors in the light curve for a particular star fails to eliminate the systematic errors in the light curve of a different star. Testing indicates each star has a small number of stars or even a single star to employ as the comparison in order to reduce the level of systematics in the light curve. On the other hand, Poisson errors in the comparison ensemble improve as the size of the comparison ensemble increases. Additionally, the volume of photometric data necessitates an automated procedure for deciding on the "best" possible comparison ensemble. Given its sensitivity to both systematic and Gaussian noise and its efficient computation, we choose to minimize the standard deviation around the mean light curve level as the figure of merit in determining the "best" comparison ensemble.

\subsection{Differential Photometry Procedure}

We balance improving systematic and Poisson errors in the light curve using the standard deviation as the figure of merit by the following procedure. The first step in determining the light curve for a star is to generate a large set of trial light curves using single comparison stars. We do not limit the potential comparison stars to the brightest or nearby stars, but calculate a light curve using all stars on the image as a potential comparison star. All comparison stars have measured photometry on at least $80 \%$ of the total number of images. A sorted list of the standard deviation around the mean light-curve level identifies the stars with the best potential for inclusion in the comparison ensemble. Calculation of the standard deviation of a light curve involves 3 iterations eliminating 3-standard-deviation outliers between iterations. However, the eliminated measurements not included in calculation of the standard deviation remain in the final light curve.

Beginning with the comparison star that resulted in the smallest standard deviation we continue to add in comparison stars with increasingly larger standard deviations. At each epoch, we median combine the results from all the comparison stars making up the ensemble after removing the average magnitude difference between target and comparison. We progressively increase the number of stars in the comparison ensemble to a maximum of 30, calculating the standard deviation of the light curve between each increase in the size of the comparison ensemble. The final light curve is determined using the comparison ensemble size that minimizes the standard deviation. Less than $1 \%$ of the stars result in the maximum of 30 comparison stars. The median number of comparison stars is 4 , with a modal value of 1 . The distribution of comparison stars has a standard deviation around the median of 4 . The fact that the standard deviations of the majority of stars is minimized using a single comparison star emphasizes the importance of considering all stars as possible comparisons in order to minimize systematic errors and achieve the highest possible accuracy.

\subsection{Comparison to a Similar Algorithm}

Independent of this study, Kovács et al. (2005) developed a generalized algorithm for eliminating systematic errors in light curves that shares several basic properties with the method we have just presented. They agree with the conclusion that optimal selection of comparison stars can eliminate systematics in the light curve. They also use the standard deviation of the light curve as their figure of merit (see their Equation 2). However, their more general implementation allows for the comparison star to have a real-valued, linear correlation coefficient $\left(c_{i}\right.$ in their Equation 1) in the differential photometry, whereas the implementation outlined here forces binary values, 0 or 1 , for the linear correlation coefficient. They solve for the real-valued, linear correlation coefficients by minimization of the standard deviation via matrix algebra, whereas the method given here relies on brute force minimization of the standard deviation. 
A thorough comparison of the performance between these methods has not been done. However, we emphasize that our algorithm found that the modal number of stars in the comparison ensemble is a single star. Their algorithm restricts the comparison ensemble to a subset of the available stars. The restricted comparison ensemble may not capture the systematics present in a light curve. However, their real-valued, linear correlation coefficients may provide the degree of freedom lacking in the algorithm of this study necessary to cancel the systematics. Both algorithms possess an important caveat. The figure of merit cannot distinguish between improvements in the Poisson error or systematic error and therefore does not guarantee optimal elimination of the systematic deviations.

\subsection{Additional Light-curve Corrections}

Although our procedure for optimally choosing comparison stars succeeds in dramatically reducing systematics in the light curves, we find that some additional systematic effects nevertheless remain. We introduce several additional corrections to the light curves to attempt to further reduce these effects.

In good seeing, brighter stars display saturation effects. Whereas, in the worst seeing, some stars display light-curve deviations that correlate with the seeing. To correct for these effects, we fit a two-piece, third-order polynomial to the correlation of magnitude versus seeing. The median seeing separates the two pieces of the fit. We first fit the good-seeing piece with the values of the polynomial coefficients unconstrained. We then fit the poor-seeing piece, but constrain the constant term such that the fit is continuous at the median seeing. However, we do not constrain the first or higher order derivatives to be continuous. In performing this fit, we excise measurements from the light curve that would lead to a seeing-correlation correction larger than the standard deviation of the light curve. We use this two-piece fit to correct the measurements.

Measurements nearby bad columns on the detector also display systematic errors that are not removed by the differential photometry algorithm. Thus, measurements when the stellar center is within 6 pixels of a bad column on the detector are eliminated from the light curve.

The final correction of the data consists of discarding measurements that deviate by more than 0.5 mag from the average light curve level. This prevents detection of companions with radii $>3.5 R_{J}$ around the lowest mass stars of the sample.

\subsection{Light-curve Noise Properties}

Figure 1 shows the logarithm of the standard deviation of the light curves as a function of the apparent $I$-band magnitude. Calculation of the standard deviation includes one iteration with 3-standard-deviation clipping. To maintain consistent $\mathrm{S} / \mathrm{N}$ at fixed apparent magnitude, the transformation between instrumental magnitude to apparent $I$-band magnitude only includes a zero-point value, since including a color term in the transformation results in stars of varying spectral shape and thus varying $\mathrm{S} / \mathrm{N}$ in the instrumental $I$ band having the same apparent $I$-band magnitude. Each individual CCD in the $8 \mathrm{~K}$ mosaic has its own zero point, and the transformation is accurate to 0.05 mag.

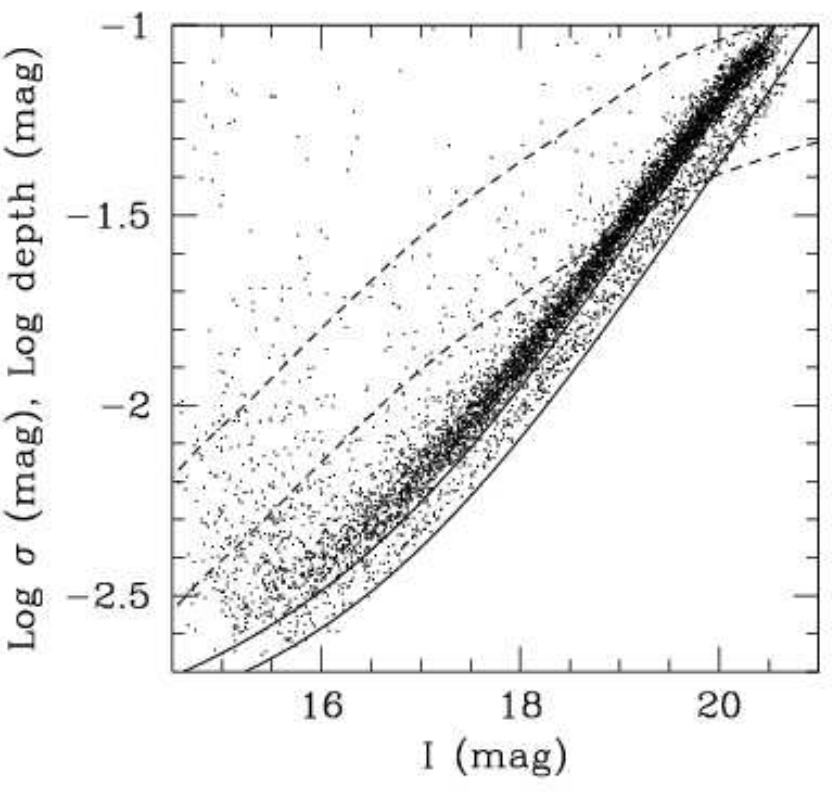

FIG. 1.- Shows the logarithm of the light-curve standard deviation as a function of the apparent $I$-band magnitude (points). The depths of transits due to a 1.0 and $1.5 R_{J}$ companion assuming the star is a cluster member are shown as dashed lines. The solid lines show photometric noise models that match the empirically determined noise properties.

One CCD has significantly better noise properties than the others as evidenced by the second sequence of points with improved standard deviation at fixed magnitude. The instrument contains a previously unidentified problem with images taken in the binning mode. The data was taken with $2 \times 2$ native pixels of the CCD array binned to one pixel on readout. During readout, the control system apparently did not record all counts in each of the four native pixels. However, the single CCD with improved noise properties does not suffer from this problem whereas all the other CCDs do. Subsequent observations with large positional shifts allow photometric measurements of the same set of stars on the affected detectors and unaffected detector. Performing these observations in the unbinned and binning modes confirms that on the affected detectors, $50 \%$ of the signal went unrecorded by the data system. This effectively reduces the quantum efficiency by half during the binned mode of operation for seven of the eight detectors.

The two solid lines outlining the locus of points in Figure 1 provide further evidence for the reduction in quantum efficiency. These lines represent the expected noise due to a a source-noise limited error, a term that scales as a background-noise limited error, and 0.0015 mag noise floor. We determine the lower line by varying the area of the seeing disk and the flat noise level until the noise model visually matches the locus of points for the detector with the lower noise properties. Then, the upper line results from assuming half the quantum efficiency of the lower noise model while keeping the noise floor the same. The excellent agreement between the higher noise model and the noise properties of the remaining detectors strongly supports the conclusion that half of 
the native pixels are not recorded during readout. This readout error could introduce significant errors in the limit of excellent seeing. However, only $4 \%$ of the photometric measurements have FWHM $<2.5$ binned pixels. Thus, even in the binning mode, we maintain sufficient sampling of the PSF to avoid issues resulting from the readout error.

The different noise properties between detectors does not complicate the analysis. The transit detection method involves $\chi^{2}$ merit criteria (see 4.2 ) that naturally handle data with varying noise properties. Other than reducing the overall effectiveness of the survey, the different noise properties between the detectors does not adversely affect the results in any way.

In addition to the empirically determined noise properties, DoPhot returns error estimates that on average result in reduced $\chi^{2}=0.93$ for a flat light-curve model. The average reduced $\chi^{2}$ for all the detector agree within $10 \%$. Scaling errors to enforce reduced $\chi^{2}=1.0$ for each detector independently has a negligible impact on the results, thus we choose not to do so.

The upper and lower dash lines in Figure 1 show the transit depth assuming the star is a cluster member for 1.5 and $1.0 R_{J}$ companions, respectively. In Figure 1] 3671 stars have light curves with a standard deviation less than the signal of a transiting $R_{J}$ companion.

\section{TRANSIT DETECTION}

In the previous section, we describe a procedure for generating light curves that reduces systematic errors that lead to false-positive transit detections. However, systematics nevertheless remain that result in highly significant false-positive transit detections. This section describes the algorithm for detecting transits and methods for eliminating false positives based on the detected transit properties. There are two types of false-positives we wish to eliminate. The first is false-positive transit detections that result from systematic errors imprinted during the signal recording and measurement process. The second type of false-positive results from true astrophysical variability that does not mimic a transit signal. For example, sinusoidal variability can result in highly significant detections in transit search algorithms. We specifically design the selection criteria to trigger on transit photometric variability that affects a minority of the measurements and that are systematically faint. However, the selection criteria do not eliminate false-positive transit signals due to true astrophysical variability that mimic the extrasolar planet transit signal we seek (grazing eclipsing binaries, diluted eclipsing binaries, etc.).

For detecting transits we employ the box-fitting least squares (BLS) method of Kovács et al. (2002). Given a trial period, phase of transit, and transit length, the BLS method provides an analytic solution for the transit depth. We show in the appendix the equivalence of the BLS method to a $\chi^{2}$ minimization. Instead of using the Signal Residue (Equation 5 in Kovács et al.|2002) or Signal Detection Efficiency (Equation 6 in Kovács et al. 2002) for quantifying the significance of the detection, we use the resulting improvement in $\chi^{2}$ of the solution relative to a constant flux fit, as outlined in the appendix.

This section begins with a discussion of the parameters affecting the BLS transit detection algorithm. We set the BLS algorithm parameters by balancing the needs of detecting transits accurately and of completing the search efficiently. The next step involves developing a set of selection criteria that automatically and robustly determines whether the best-fit transit parameters result from bona fide astrophysical variability that resembles a transit signal. A set of automated selection criteria that only pass bona fide variability is a critical component of analyzing the null-result transit survey and has been ignored in previous analyses.

Due to the systematic errors present in the light curve, statistical significance of a transit with a Gaussian noise basis is not applicable. In addition, the statistical significance is difficult to calculate given the large number of trial phases, periods, and inclinations searched for transits. Given these limitations, we empirically determine the selection criteria on the actual light curves. Although it is impossible to assign a formal false alarm probability to our selection criteria, the exact values for the selection criteria are not important as long as the cuts eliminate the false positives while still maintaining the ability to detect $R_{J}$ objects, and identical criteria are employed in the Monte Carlo detection probability calculation.

\subsection{BLS Transit Detection Parameters}

The BLS algorithm has two parameters that determine the resolution of the transit search. The first parameter determines the resolution of the trial orbital periods. The BLS algorithm (as implemented by Kovács et al. 2002) employs a period resolution with even frequency intervals, $\frac{1}{P_{2}}=\frac{1}{P_{1}}-\eta$, where $P_{1}$ is the previous trial orbital period, $P_{2}$ is the subsequent (longer) trial orbital period, and $\eta$ determines the frequency spacing between trial orbital periods. During implementation of the BLS algorithm, we adopt an even logarithmic period resolution by fractionally increasing the period, $P_{2}=P_{1} \times(1+\eta)$. The original implementation by Kovács et al. (2002) for the orbital-period spacing is a more appropriate procedure, since even frequency intervals maintain constant orbital phase shifts of a measurement between subsequent trial orbital periods. The even logarithmic period resolution we employ results in coarser orbital phase shifts between subsequent trial orbital periods for the shortest periods and increasingly finer orbital phase shifts toward longer trial orbital periods. Either period-sampling procedure remains valid with sufficient resolution. We adopt $\eta=0.0025$, which, given the observational baseline of 19 days, provides $<10 \%$ orbital phase shifts for orbital periods as short as 0.5 day.

The second parameter of the BLS algorithm determines the resolution in orbital phase by binning the phase-folded light curve. Binning of the data in orbital phase drastically improves the numerical efficiency, but not without loss in determining the correct transit properties. Kovács et al. (2002) give a thorough examination of how the sensitivity in recovering transits varies with orbital-phase binning resolution. To search for transit candidates in the light curves we adopt $N_{\text {bins }}=400$ orbital-phase bins. We verify with tests that the above parameters accurately recover boxcar signals in the light curves. After injection of boxcar signals in the light curves, we calculate the $\chi^{2}$ of the solution returned by the BLS method with the $\chi^{2}$ of the injected model. Tests show that the BLS method with the above parameters return a $\chi^{2}$ within $30 \%$, and typically much better, of 
the injected model's $\chi^{2}$.

\subsection{Selection Criteria}

We apply the BLS method following the description in the previous section to search for transit candidates in all 6787 stars with light curves. A visual inspection of a light curve folded at the best-fit transit period can generally be used to discriminate between bona fide astrophysical variability and a false positive arising from systematic errors. However, a proper statistical assessment of the sensitivity of a transit search requires that the exact same set of selection criteria that are applied to cull false positives are also applied when assessing the detection probability via, e.g., Monte Carlo injection and recovery of artificial signals. Due to the large number of artificial signals that must be injected to calculate the detection probability properly, using selection criteria based on visual inspection of light curves is practically very difficult or impossible. Therefore, quantitative, automated detection criteria that mimic the visual criteria must be used.

We employ four selection criteria that eliminate all false detections while still maintaining the ability to detect $R_{J}$ companions. These four selection criteria constitute cuts on (1) the improvement in $\chi^{2}$ of a transit model over a constant flux model, (2) the ratio between the $\Delta \chi^{2}$ of the best-fit transit model and the $\Delta \chi_{-}^{2}$ of the best-fit anti-transit model, (3) the fraction of $\Delta \chi^{2}$ from a single night, and (4) the transit period.

The first of the selection criteria is a cut on $\Delta \chi^{2}$, the improvement in $\chi^{2}$ between a constant flux model and a transit model. The $\Delta \chi^{2}$ is similar to the Signal Residue, $\mathrm{SR}$, of Kovács et al. (2002); we derive $\Delta \chi^{2}$ and its relation to $\mathrm{SR}$ in the appendix. We prefer $\Delta \chi^{2}$ over SR as the former allows a direct comparison of the transit detection significance between light curves with different noise properties. Given the correlated systematics in the data, we cannot rely on analytical formulations with a Gaussian statistics basis for the statistical significance of a particular $\Delta \chi^{2}$ value. We empirically determine a cut on $\Delta \chi^{2}$ in combination with the other selection criteria to fully eliminate false detections. For a transit detection we require $\Delta \chi^{2}>95.0$. As shown in the appendix, this selection criterion corresponds to a $\mathrm{S} / \mathrm{N} \sim 10$ transit detection. Figure 2 shows the $\Delta \chi^{2}$ of the best fit transit for all light curves along the x-axis. The vertical line designates the selection criteria on this parameter. Even with such a strict threshold, there are still a large number of false positives that pass the $\Delta \chi^{2}$ cut.

Systematic variations in the light curves that are characterized by small reductions in the apparent flux of star that are coherent over the typical time scales of planetary transits can give rise to false-positive transit detections. However, under the reasonable expectation that systematics do not have a strong tendency to produce dimming versus brightening of the apparent flux of the stars, one would expect systematics to also result in a false-positive 'anti-transit' (brightening) detections. Furthermore, most intrinsic variables can be approximately characterized by sinusoids, which will also result in significant transit and anti-transit detections. On the other hand, a light curve with a true transit signal and insignificant systematics should produce only a strong transit detection, and not a strong anti-transit detection.

Thus, the ratio of the significance of the best-fit tran-

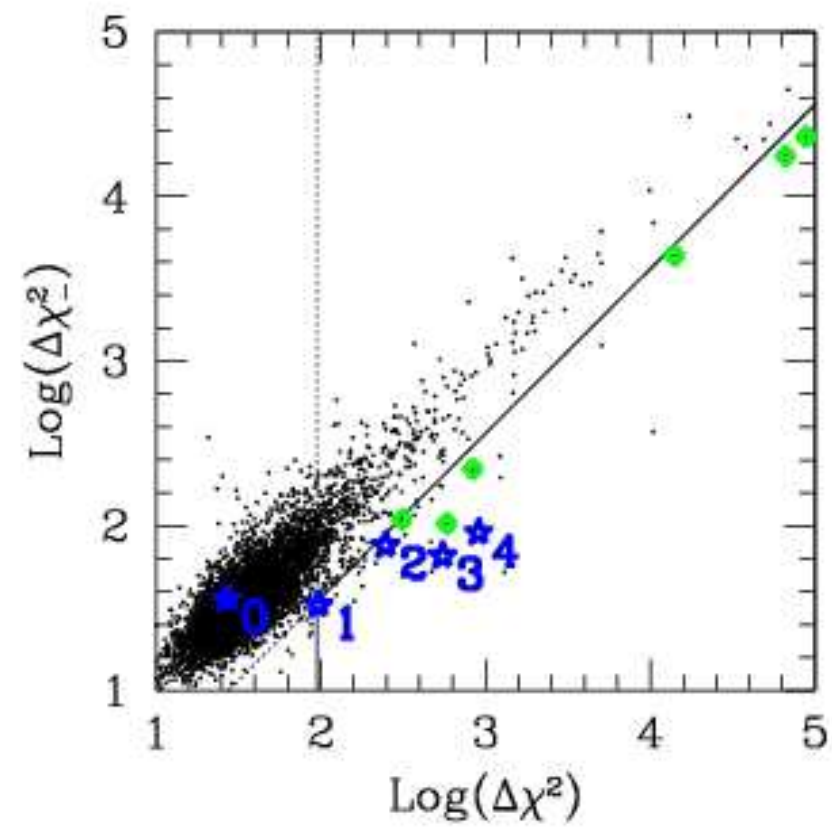

FIG. 2.- The small points show $\Delta \chi^{2}$ as a function of $\Delta \chi_{-}^{2}$ for the resulting best-fit transit parameters in all light curves. Here $\Delta \chi^{2}$ and $\Delta \chi_{-}^{2}$ are the $\chi^{2}$ improvement between the flat light-curve model and the best-fit transit and anti-transit model, respectively. The dotted vertical line shows the $\Delta \chi^{2}=95.0$ selection boundary. The solid diagonal line shows the $\Delta \chi^{2} / \Delta \chi_{-}^{2}=2.75$ selection boundary. Objects in the lower right corner pass both selection criteria. The green diamonds show values of $\Delta \chi^{2}$ and $\Delta \chi_{-}^{2}$ for the six transit candidates. The blue stars show the recovered values of $\Delta \chi^{2}$ and $\Delta \chi_{-}^{2}$ for the four light curves with injected transits shown in Figure 7 The label next to the blue stars corresponds to the label in the upper right corner of each panel in Figure 7 These curves were created by injecting transits into the same light curve. The blue star labeled 0 shows the values of $\Delta \chi^{2}$ and $\Delta \chi_{-}^{2}$ for this light curve before the example transits were injected.

sit signal relative to that of the best-fit anti-transit signal provides a rough estimate of the degree to which a detection has the expected properties of a bona fide transit, rather than the properties of systematics or sinusoidal variability. In other words, a highly significant transit signal should have a negligible anti-transit signal, and therefore we require the best-fit transit to have a greater significance than the best-fit anti-transit. We accomplish this by requiring transit detections to have $\Delta \chi^{2} / \Delta \chi_{-}^{2}>2.75$, where $\Delta \chi_{-}^{2}$ is the $\chi^{2}$ improvement of the best-fit anti-transit. For a given trial period, phase of transit, and length of transit, the BLS algorithm returns the best-fit transit without restriction on the sign of the transit depth. Thus, the BLS algorithm simultaneously searches for the best-fit transit and anti-transit, and so determining $\Delta \chi_{-}^{2}$ has no impact on the numerical efficiency.

Figure 2 shows the $\Delta \chi_{-}^{2}$ of the best fit anti-transit versus the $\Delta \chi^{2}$ of the best-fit transit for our light curves. 
The diagonal line demonstrates the selection on the ratio $\Delta \chi^{2} / \Delta \chi_{-}^{2}=2.75$. Objects toward the lower right corner of this Figure pass the selection criteria. The objects with large $\Delta \chi^{2}$ typically have correspondingly large $\Delta \chi_{-}^{2}$. This occurs for sinusoidal-like variability or strong systematics that generally have both times of bright and faint measurements with respect to the mean light-curve level.

Requiring observations of the transit signal on separate nights also aids in eliminating false-positive detections. We quantify the fraction of a transit that occurs during each night based on the fraction of the transit's $\chi^{2}$ significance that occurs during each night. The parameters of the transit allow identification of the data points that occur during the transit. We sum the individual $\chi_{i}^{2}=\left(m_{i} / \sigma_{i}\right)^{2}$ values for data points occurring during the transit to derive $\chi_{\text {tot }}^{2}$, where $m_{i}$ is the light curve measurement and $\sigma_{i}$ is its error. Then we calculate the same sum for each night individually. We denote this $\chi_{k \text { th night }}^{2}$. We identify the night for which $\chi_{k \text { th night }}^{2}$ contributes the greatest fraction of $\chi_{\text {tot }}^{2}$, and we call this fraction $f=\chi_{k \text { th night }}^{2} / \chi_{\text {tot }}^{2}$. Finally, we require $f<0.65$. This corresponds to roughly seeing the transit one and a half times assuming all observations have similar noise. Alternatively, this criterion is also met by observing $2 / 3$ of a transit on one night and $1 / 3$ of the transit on a separate night, or observing a full transit on one night and $1 / 6$ of transit on a separate night with three times improvement in the photometric error. Figure 3 shows $f$ versus the best-fit period for all the light curves. The horizontal line designates the selection on this parameter.

The red points in Figure 3 show objects that pass the $\Delta \chi^{2}>95.0$ selection. We find that most are clustered around a 1.0 day orbital period. A histogram of the bestfit transit periods amongst all light curves reveals a high frequency for 1.0 day and 0.5 day periods. Visual inspection of the phased light curves reveals a high propensity for systematic deviations to occur on the Earth's rotational period and 0.5 day alias. We do not fully understand the origin of this effect, but we can easily conjecture on several effects that may arise over the course of an evening as the telescope tracks from horizon to horizon following the Earth's diurnal motion. In order to eliminate these false positives, we apply as our fourth selection criteria a cut on the period. Specifically, we require transit detections to have periods that are not within $1.0 \pm 0.1$ and $0.5 \pm 0.025$ day. The horizontal lines designate these ranges of discarded periods.

\section{TRANSIT CANDIDATES}

Six out of 6787 stars pass all four selection criteria. All of these stars are likely real astrophysical variables whose variability resembles that of planetary transit light curves. However, we find that none are bona fide planetary transits in NGC 1245. After describing the properties of these objects we will describe the procedure for ruling out their planetary nature. Figure 4 shows the phased light curves for these six stars. Each light-curve panel in Figure 4 has a different magnitude scale with fainter flux levels being more negative. The upper left corner of each panel gives the detected transit period as given by the BLS method. The upper right corner of each panel gives an internal identification number. The panels

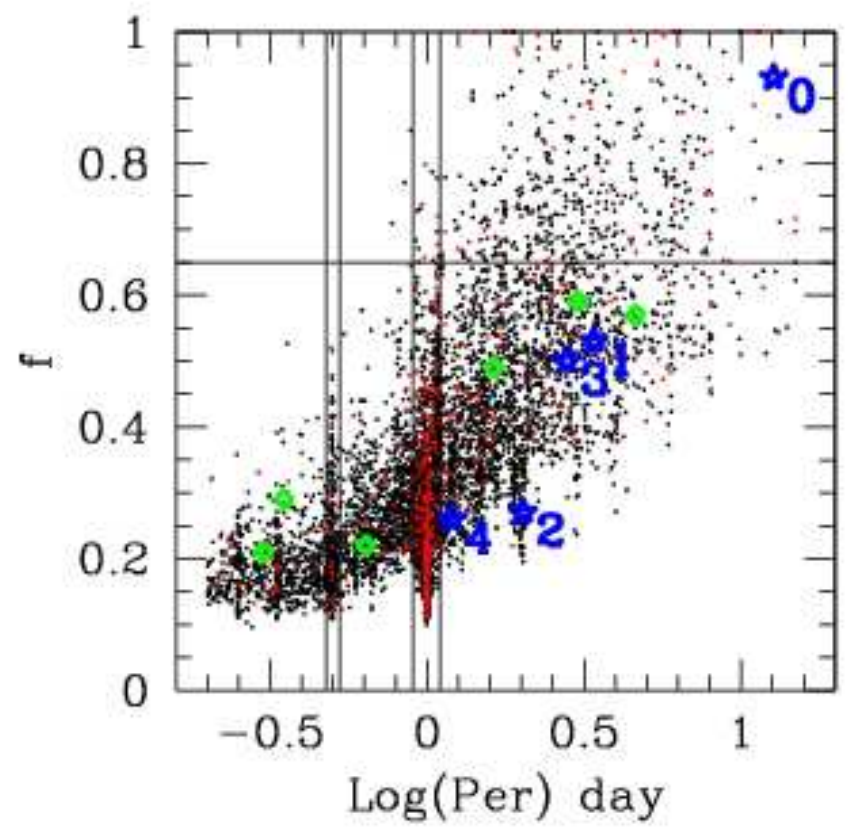

FIG. 3.- The black points show $f$ as a function of the best-fit transit orbital period, where $f$ is the fraction of the total $\chi^{2}$ improvement with the best-fit transit model that comes from a single night. The objects that pass the $\Delta \chi^{2}>95.0$ selection criteria are shown as red points. The horizontal line shows the $f=0.65$ selection boundary. The vertical lines denote orbital period regions avoided due to false-positive transit detections. The blue stars and green diamonds are the same as in Figure 2

from top to bottom have decreasing values in the ratio between the improvement of a transit and anti-transit model, $\Delta \chi^{2} / \Delta \chi_{-}^{2}$.

Table 2 lists the properties and selection criteria values for the stars shown in Figure 4 . The green diamonds in Figures 2 and 3 represent the selection criteria for the six transit candidates. The photometric and positional data in Table 2 come from Burke et al. (2004). The $\chi_{\text {mem }}^{2}$ entry in Table 2 measures the photometric distance of a star from the isochrone that best fits the cluster CMD. A lower value of this parameter means a star has a position in the CMD closer to the main sequence. Heavy points in Figure 5] denote stars with $\chi_{\text {mem }}^{2}<0.04$, and we designate these stars as potential cluster members. Based on $\chi_{\text {mem }}^{2}$, star 20513 and star 70178 have photometry consistent with cluster membership, thus we also list the physical parameters of those stars in Table 2 Burke et al. (2004) details the procedure for determining the physical parameters of a star based solely on the broad-band photometry and the best-fit cluster isochrone. However, the validity of the stellar physical parameters only applies if the star is a bona fide cluster member.

Figure 6] shows a finding chart for each star with a light curve in Figure 4 The label in each panel gives the identification number, and the cross indicates the corre- 


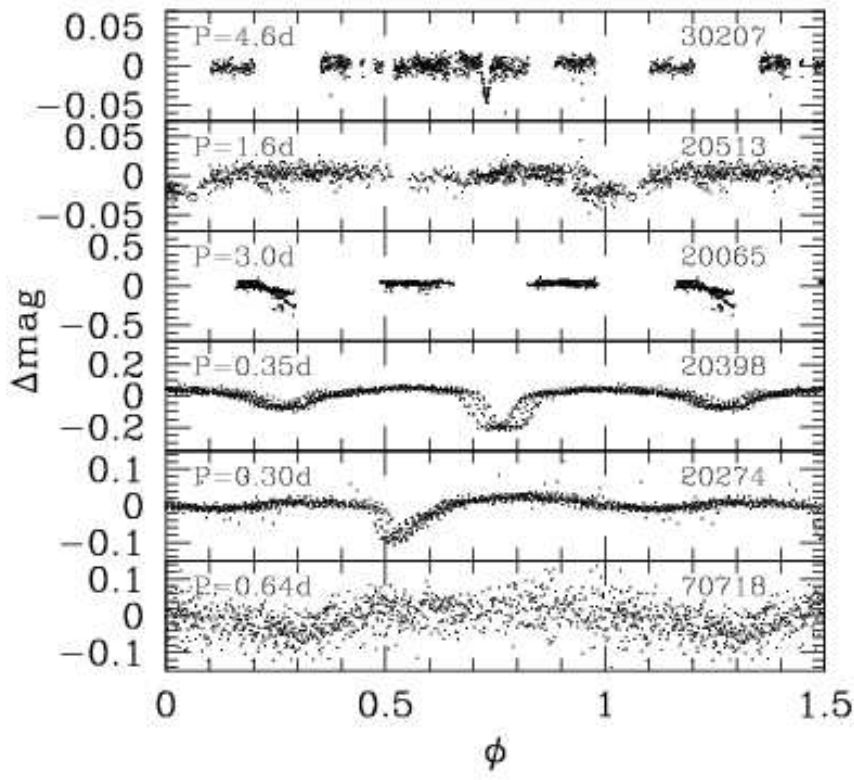

FIG. 4.- The points show the change in magnitude as a function of orbital phase for all stars that meet the transit candidate selection criteria. Negative values for $\Delta \mathrm{mag}$ are toward fainter flux levels. The phased period is given in the upper left corner of each panel, and the number in the upper right corner of each panel gives the internal identification number.

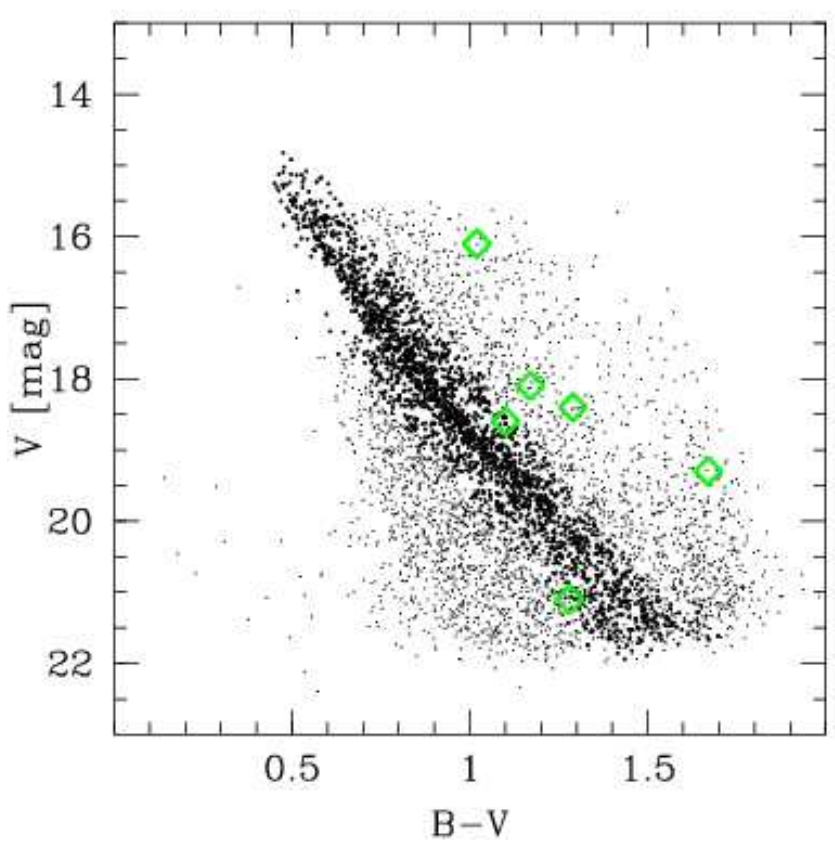

FIG. 5.- The CMD of the cluster NGC 1245. Potential cluster members having $\chi_{\text {mem }}^{2}<0.04$ are shown with heavy points). Objects that exceed the selection criteria for transit detection are given as open diamonds.

sponding object. Star 20274 is not centered in the finding chart because it is located near the detector edge. The field of view of each panel is $54^{\prime \prime}$. North is toward the right, and East is toward the bottom. The panels for stars 20065, 20398, and 20513 (located near the cluster

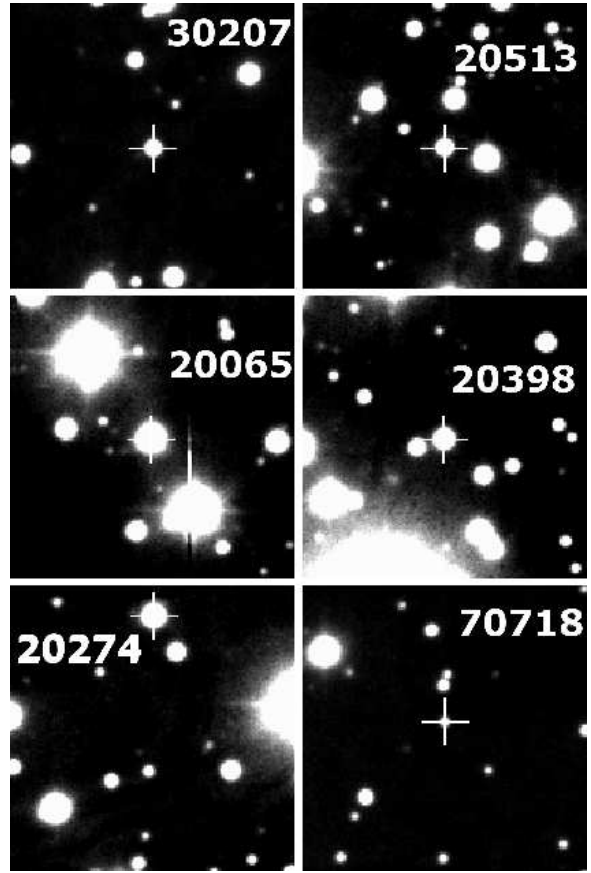

FIG. 6.- Finding charts for transit candidates with light curves shown in Figure 4 Each panel is $54^{\prime \prime}$ on a side. North is toward the right, and East is toward the bottom.

center) provide a visual impression of the heaviest stellar crowding encountered in the data. Figure 5 shows the $V$ and $B-V \mathrm{CMD}$ of the cluster field as given in Burke et al. (2004). The large open stars denote the locations of the objects that exceed the transit selection criteria.

\subsection{Consistency of Transit Parameters with Cluster Membership}

Only stars 20513 and 70718 have $\chi_{\text {mem }}^{2}$ values consistent with cluster membership. Additionally, the transit depth in both stars indicates potential for having a $R_{J}$ companion. However, qualitatively, in each case the transit duration relative to the orbital period is too long to be a true planetary companion to a cluster main-sequence star. We can use our knowledge of the physical properties of the parent stars to quantitatively rule out planetary companions. We do this by comparing an estimate of the stellar radius derived from the CMD to an independent estimate of a lower limit on the stellar radius derived from the properties of the light curve. In both cases we find that the stellar radii derived from the CMD are well below the lower limit on the stellar radius based on the light curve.

To derive a lower limit on the stellar radius from the light curve, we build on the work by Seager \& Mallén-Ornelas (2003). They provide a purely geometric relationship between the orbital semimajor axis, $a$, and stellar radius, $R_{\star}$, for a light curve with a given period, $P$, depth of transit, $\Delta F$, and total duration of the transit (first to fourth contact), $\tau$, assuming a circular orbit (see their Equation 8). By assuming a central transit (impact parameter $b=0$ ), we transform their equality into a lower limit. Using Kepler's Third Law, assuming that the mass of the companion is much smaller 
than the mass of the star, and assuming the duration of the transit is much smaller than the period $(\tau \ll P)$, we find,

$$
R_{\star}>\frac{\pi\left(M_{\star}+m_{p}\right)^{1 / 3} \tau}{P^{1 / 3}(1+\sqrt{\Delta F})} .
$$

where $R_{\star}$ is in $\mathrm{AU}, M_{\star}$ is in units of $M_{\odot}$ and $\tau$ and $P$ are in years.

Parameters on the right hand side of the above equation contain substantial uncertainties. Replacing the parameters by their maximum plausible deviation from their measured values in such a manner as to decrease $R_{\star}$ increases the robustness of the lower limit. The orbital period determination has the largest uncertainty. Tests of recovering transits in the light curves reveal a $10 \%$ chance for the BLS method to return an orbital period, $P^{\prime}$, at the $1 / 2 P$ and $2 P$ aliases of the injected orbital period, and a $<1 \%$ chance of detecting the $1 / 3 P$ and $3 P$ aliases. Misidentification of the correct orbital period results from gaps in the observing window function. Replacing $P$ in the above equation with $3 P^{\prime}$, where $P^{\prime}$ is the orbital period returned by the BLS algorithm, provides the maximum plausible deviation of this quantity and increases the robustness of the lower limit. In addition, the stellar mass determination based on the CMD potentially has contamination from a binary companion. Thus, we replace $M_{\star}$ with $0.5 M_{\star}^{\prime}$, where $M_{\star}^{\prime}$ is the stellar mass estimate from the CMD. We do not modify $\tau$ or $\Delta F$. For the cases considered here, $\Delta F \ll 1$, and the term $1+\sqrt{\Delta F} \simeq 1$ in Equation 11 Therefore the precise value of $\Delta F$ has little effect on the resulting limit on $R_{*}$. The BLS algorithm fits a boxcar transit model to the light curve via a $\chi^{2}$ minimization. Since, in the limit of zero noise, any non-zero boxcar height fit to a transit can only result in an increasing $\chi^{2}$ when the length of the boxcar exceeds the length of the transit, $\tau$ underestimates the true transit length. Making the above replacements the lower limit on the stellar radius is,

$$
R_{\star}>7.3 \frac{\left(M_{\star}^{\prime} / M_{\odot}\right)^{1 / 3}(\tau / 1 \text { day })}{\left(P^{\prime} / 1 \text { day }\right)^{1 / 3}(1+\sqrt{\Delta \mathrm{F}})} R_{\odot} .
$$

For star 20513, the above equation requires $R_{\star}>$ $1.04 R_{\odot}$ if the star is a cluster member. Fits to the CMD yield a stellar radius $R_{\star}=0.80 R_{\odot}$. The lower limit for star 70718 is $R_{\star}>0.82 R_{\odot}$, whereas the CMD yields $R_{\star}=0.56 R_{\odot}$. Clearly both stars lack consistency between the stellar radius based on the CMD location and the stellar radius based on the transit properties.

The transiting companions to 20513 and 70718 are also unlikely to be planets if the host stars are field dwarfs. Tinglev \& Sackett (2005) provide a diagnostic to verify the planetary nature of a transit when only the light curve is available. The diagnostic $\eta_{p}$ of Tinglev \& Sackett (2005) compares the length of the observed transit to an estimate of the transit length derived by assuming a main-sequence mass-radius relation for the central star. By assuming a radius of the companion of $R_{p}=1.0 R_{J}$, we find $\eta_{p}=4.0$ and 3.8 for 20513 and 70718 , respectively. Values of $\eta_{p} \lesssim 1$ correspond to planetary transits. Therefore, 20513 and 70718 are unlikely to host planetary companions with $R_{p} \lesssim R_{J}$ if they are main-sequence stars.

We note that our final a posteriori criterion with which we reject cluster transit candidates, namely the consis- tency between the radius of the parent star as estimated from the CMD and the radius as estimated from the light curve, is a conceptually different kind of selection criterion than those that we applied to all the light curves to arrive at our six transit candidates. The original four selection criteria were designed to detect bona fide astrophysical variability that resembles the signals from transiting planets, but does not necessarily arise from a transiting planetary companion. In principle, we could have included the radius consistency cut as an additional selection criterion applied to all light curves. The motivation to do this would be that imposing this additional criterion might automatically remove some systematic false positives and so allow us to improve our efficiency by making the other selection criteria less stringent. We have found using limited tests that this is not the case. We therefore chose to leave the radius check as an a posteriori cut on the transit candidates. Nevertheless, observing a cluster does provide an advantage over observing field stars, as the additional constraint on the stellar radius from the cluster CMD provides a more reliable confirmation of the planetary nature than the light curve alone (Tinglev \& Sackett 2005), and furthermore allows a more accurate assessment of the detection probability.

It is important to emphasize that all of the injected transits with which we compute the detection probability (§ 6) automatically pass the radius consistency criterion. A fraction of these will be recovered at periods that differ enough from the input period that by using the recovered period they will no longer satisfy the radius constraint. However, we find that this fraction is negligibly small.

\subsection{Individual Cases}

This section briefly discusses each object that met the selection criteria as a transit candidate but does not belong to the cluster. The V-shaped transit detected in star 30207 rules out a $R_{J}$ companion. Transiting $R_{J}$ companions result in a flat bottomed eclipse as the stellar disk fully encompasses the planetary disk. A closer inspection of the light curve also reveals ellipsoidal variations outside of the transit. This light curve matches the properties of a grazing eclipse, which is a typical contaminant in transit searches (e.g., Bouchv et al. 2005).

The remaining stars have depths too large for a $R_{J}$ companion and show evidence for secondary eclipses. Recall that we eliminated data points with $|\Delta m|>0.5 \mathrm{mag}$ in the light curves. This eliminates the eclipse bottom for star 20065. Keeping all the data for star 20065 clearly reveals the characteristics of a detached eclipsing binary. The period BLS derives for star 20065 aligns the primary and secondary eclipses, and thus BLS-reported period is not the true orbital period.

The eclipses in stars 20398 and 20274 do not perfectly phase up. This is because the resolution in period we used for the search prevents perfect alignment of the eclipses for such short periods. This effect is inconsequential for detecting transiting planets as they all have orbital periods longer than 0.3 day.

Finally, we note that other variables exist in the dataset. They were not selected because they do not meet the $\Delta \chi_{\min }^{2} / \Delta \chi_{\min }^{2}$ - selection criterion. A future paper will present variables that exist in this dataset using selection criteria more appropriate for identifying quasi-sinusoidal periodic variability (J. Pepper et al., in 
preparation).

\section{DETECTION PROBABILITY CALCULATION}

We did not detect any transit signals consistent with a $R_{J}$ companion. To interpret this null result in terms of the frequency of planetary companions to stars in NGC 1245, we develop a Monte Carlo detection probability calculation for quantifying the sensitivity of the survey for detecting extrasolar planet transits. The calculation provides the probability of detecting a transit in the survey as a function of the companion semimajor axis and radius. In addition to the photometric noise and observing window, the observed properties of the transit signal depend sensitively on the host mass, radius, limbdarkening parameters, and orbital inclination with respect to the line of sight. Without accurate knowledge of the stellar parameters, a detailed detection probability is not possible. This precludes analyzing stars not belonging to the cluster. Given the degeneracy between broad-band colors of dwarfs, subgiants, and giants, the stellar radius for most field objects cannot be determined from the CMD alone. Assuming all stars of a given color are dwarfs drastically overestimates the number of actual dwarf stars in a transit survey (Gould \& Morgan 2003). The minimal expenditure of observational resources necessary for determining the stellar parameters for a cluster transit survey provides a significant advantage over transit surveys of the field.

Each star in the survey has a unique set of physical properties and photometric noise, thus we calculate the detection probability for all stars in the survey. This is the first study of its kind to do so. Given the detection probability for each star, the distribution of extrasolar planet semimajor axis, and frequency of extrasolar planet occurrence, the survey should have detected,

$$
N_{\text {det }}=f_{\star} \sum_{i=1}^{N_{\star}} P_{\text {det }, \mathrm{i}}
$$

extrasolar planets, where the sum is over all stars in the survey,

$$
P_{\mathrm{det}, \mathrm{i}}=\iint \frac{d^{2} p}{d R_{p} d a} P_{\epsilon, i}\left(a, R_{p}\right) P_{T, i}\left(a, R_{p}\right) P_{\mathrm{mem}, \mathrm{i}} d R_{p} d a,
$$

$R_{p}$ is the extrasolar planet radius, $a$ is the semimajor axis, $f_{\star}$ is the fraction of stars with planets distributed according to the joint probability distribution of $R_{p}$ and $a, \frac{d^{2} p}{d R_{p} d a}$. The Monte Carlo detection probability calculation provides $P_{\epsilon, i}\left(a, R_{p}\right)$, the probability of detecting a transit in a given light curve. The term $P_{T, i}\left(a, R_{p}\right)$ gives the probability for the planet to cross the limb of the host along the line of sight, and $P_{\text {mem,i }}$ gives the probability the star is a cluster member. This framework for calculating the expected detections of the survey follows from the work of Gaudi et al. (2002). In the following subsections we describe the procedure for calculating each of these probability terms.

\subsection{Calculating $P_{\epsilon, i}\left(a, R_{p}\right)$}

$P_{\epsilon, i}\left(a, R_{p}\right)$ is the probability of detecting a transit around the $i$ th star of the survey averaged over the orbital phase and orbital inclination for a given companion radius and semimajor axis. We begin this section with a description of the procedure for injecting limb-darkened transits into light curves for recovery. After injecting the transit, we attempt to recover the transit employing the same BLS algorithm and selection criteria as employed during the transit search on the original data. It is critical to employ identical selection criteria during the recovery as the original transit search since only then can we trust the robustness and statistical significance of the detection. The fraction of transits recovered for fixed semimajor axis and $R_{p}$ determines $P_{\epsilon}$. Next, we characterize the sources of error present in $P_{\epsilon}$ and how we ensure a specified level of accuracy. Finally, in this section we discuss the parallelization of the calculation to obtain $P_{\epsilon}$ for all stars in the survey in a reasonable amount of time.

In the appendix, we discuss the importance of injecting realistic transits for recovery. Mandel \& Agol (2002) provide analytic formulas for calculating realistic limbdarkened transits. We employ the functional form of a transit for a quadratic limb-darkening law as given in Section 4 of Mandel \& Agol (2002). The quadratic limbdarkening coefficients come from Claret (2000). Specifically, we use the $I$-band limb-darkening coefficients using the ATLAS calculation for $\log g=4.5, \log [\mathrm{M} / \mathrm{H}]=0.0$, and $v_{\text {turb }}=2 \mathrm{kms}^{-1}$.

We assume circular orbits for the companions. All known extrasolar planets to date that orbit within 0.1 $\mathrm{AU}$ have eccentricities $<0.3$, and the average eccentricity for these planets is $\langle e\rangle=0.07^{6}$.

After injecting the transit, we employ the BLS algorithm to recover the injected transit signal using the selection criteria described in 4.2 For numerical efficiency, we relax the resolution of the BLS search parameters. We adopt a fractional period step, $\eta=0.004$, and phase space binning, $N_{\text {bins }}=300$. Despite the reduced resolution, higher resolution, converged solutions reveal only a 0.003 lower probability resulting from the adopted parameters. We correct all probabilities for this systematic even though it is at an insignificant level compared to the other uncertainties.

Figure 7 visualizes the injected transits with increasing degrees of significance from top to bottom. This Figure shows light curves with an injected transit phased at the period as returned from the BLS algorithm. The solid line illustrates the injected limb-darkened transit signal. The top two panels and the bottom two panels illustrate 1.0 and $1.5 R_{J}$ companions, respectively. The transit recovery in the top panel barely meets the selection criteria, thus giving a visual impression for the sensitivity of the survey. The resulting selection criteria values after recovery of the injected transits are shown in Figures 2 and 3 by the blue stars, and the labels next to the stars correspond to the panel label given in the upper right hand corner. The modeled transits shown in this Figure are injected into the same light curve of a potential cluster member with $V=16.6$ and rms scatter (before transit injection) of $\sigma=0.003$. The blue star, labeled 0 in Figures 2 and 3. represents the values for the selection criteria for found for this light curve before injecting the transits.

As opposed to previous work, we carefully examine,

${ }^{6}$ http://www.obspm.fr/encycl/catalog.html 


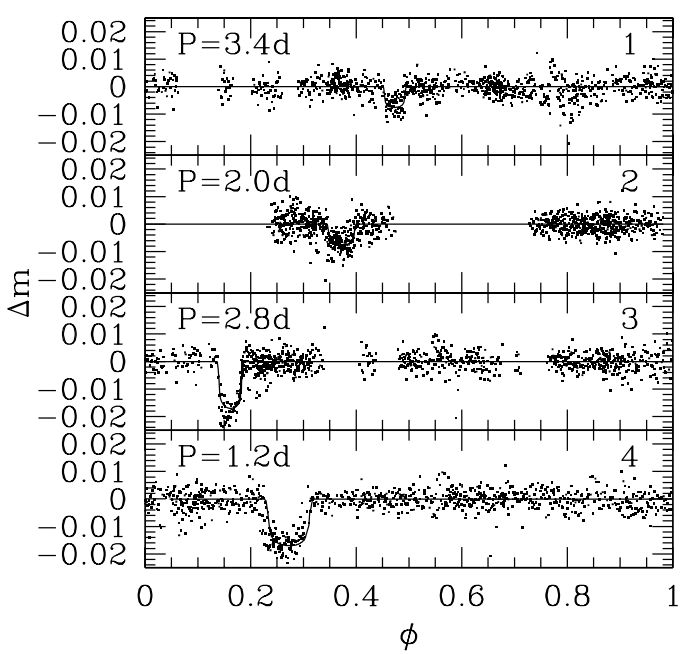

FIG. 7.- Phased light curves showing the recovery of transits injected in the light curve by the Monte Carlo calculation. The injected limb-darkened transit signal is given by the solid line. The top two panels and bottom two panels show results for 1.0 and 1.5 $R_{J}$ companions, respectively. The transit recovery in the top panel barely meets the selection criteria and gives an impression for the sensitivity of the survey. The labels in the upper right corner of the panels correspond to the markers for the selection criteria values shown in Figures 2 and 3

quantify, and control the uncertainties present in the calculation. During injection of a transit at fixed semimajor axis, the transit can occur during any phase of the orbit. We use the following procedure to ensure that we inject enough trial transits at random orbital phases to yield convergence of $P_{\epsilon}$. Based on binomial statistics, the error in the resulting probability at fixed orbital period depends on the actual probability and the number of trial transit phases, $\sigma_{\epsilon}=\sqrt{N_{\text {trial }} \epsilon_{\text {act }}\left(1-\epsilon_{\text {act }}\right)}$, where $N_{\text {trial }}$ is the number of trial transit phases and $\epsilon_{\text {act }}$ is the actual probability (unknown a priori). Maintaining the same error in the detection probability for differing $\epsilon_{\text {act }}$ requires a variable number of trial phases. For each semimajor axis, we first obtain an initial estimate for the probability, $\epsilon_{\text {est }}$, using $N_{\text {trial }}=100$. We then increase $N_{\text {trial }}$ until the probability converges to $\sigma_{\epsilon}=\sqrt{N_{\text {trial }} \epsilon_{\text {est }}\left(1-\epsilon_{\text {est }}\right)} \leq 0.02$. The above procedure systematically overestimates $\epsilon_{\text {act }}$ when $\epsilon_{\text {act }} \gtrsim 0.95$ and systematically underestimates $\epsilon_{\text {act }}$ when $\epsilon_{\text {act }} \lesssim 0.05$. However, these errors are of order the adopted $\sigma_{\epsilon}=0.02$ accuracy, and so we neglect them here.

In addition to a random orbital phase, assuming a random orientation of the orbit requires taking into account an even distribution in $\cos i$, where $i$ is the inclination of the orbit. Only a narrow range of inclinations, $\cos i \leq\left(R_{\star}+R_{p}\right) / a$, results in a transit. Thus, we inject the transit with an even distribution in $\cos i$ between $0 \leq \cos i \leq\left(R_{\star}+R_{p}\right) / a$.

The previous discussion pertains to ensuring a prescribed accuracy at fixed semimajor axis. However, the expected detection rate also requires an integral over semimajor axis, which must be sampled at high enough resolution to ensure convergence of the integral. We calculate the probability at even logarithmic intervals, $\delta \log a=0.011$ AU. In comparison to high-resolution, converged calculations, this semimajor axis resolution results in an absolute error in the detection probability integrated over the semimajor axis of $\sigma_{\epsilon}=0.003$. We inject transits with semimajor axis from the larger of $0.0035 \mathrm{AU}$ and $1.5 R_{\star}$ to $0.83 \mathrm{AU}$. The best-fit isochrone to the cluster CMD determines the parent star radius.

Generating the light curve from the raw photometric measurements is numerically time consuming. Thus, we inject the transit after generating the light curve. This procedure has the potential to systematically reduce or even eliminate the transit signal, because generating the light curve and applying a seeing decorrelation tend to "flatten" a light curve. To quantify the significance of this effect, we inject transits in the raw photometric measurements before the light curve generation procedure on several stars in the sample. Comparing the detection probability obtained by injecting transits before light curve generation to the detection probability obtained by injecting the transit after light curve generation reveals that injecting the transit after generating the light curve overestimates the detection probability by $\sim 0.03$. We decrease the calculated probability at fixed period by 0.03 to account for this systematic effect.

The 0.03 systematic overestimate in the detection probability becomes increasingly important for correctly characterizing the detection probability at long orbital periods. For instance, the detection probability for a star of median brightness will be overestimated by $>15 \%$ for orbital periods $>4.0$ day and $1.5 R_{J}$ companions if this systematic effect is not taken into account. The detection probability is overestimated by $>50 \%$ for orbital periods $>8.0$ day without correction. The results for $1.0 R_{J}$ companions are even more severe. The detection probability would be overestimated by $50 \%$ for periods beyond 1.8 day for a star of median brightness without correction.

Based on the CMD of NGC 1245 (Burke et al. 2004), this study contains light curves for $\sim 2700$ stars consistent with cluster membership. Initially, we calculate the detection probability for 2 possible companion radii: 1.0 and $1.5 R_{J}$. For each star, on average we inject 50000 transits for a single companion radius at 150 different semimajor axes. In total, we inject and attempt to recover $\sim 2.7 \times 10^{8}$ transits. Current processors allow injection and attempted recovery on order of $1 \mathrm{~s}$ per transit. A single processor requires $\sim 3000$ days for the entire calculation. Fortunately, the complete independence of a transit injection and recovery trial allows parallelization of the calculation. We accomplish a parallel calculation via a server and client architecture. A server injects a transit in the current light curve and sends it to a client for recovery.

Based on the computing resources available, we employ two different methods for communication between the server and clients. Using a TCP/IP UNIX socket implementation for communication between the server and clients allows access to $\sim 40$ single-processor personal workstations connected via a local area network within the department of astronomy at The Ohio State University. Additionally, the department of astronomy at The Ohio State University has exclusive access to a 48 processor Beowulf cluster via the Cluster Ohio program run by the Ohio Supercomputer Center. The Message Passing Interface (MPI) libraries provide communication 


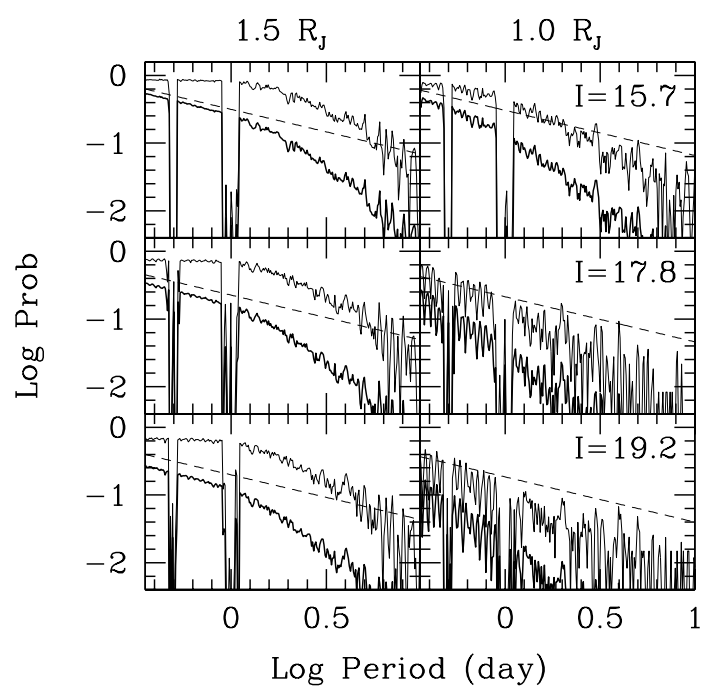

FIG. 8.- Detection probability as a function of the orbital period is shown as the heavy solid line. This is a product of the probability for a transit to occur (dash line) and the probability that an injected transit meets the selection criteria (light solid line). The panels from top to bottom show representative stars in order of increasing apparent magnitude. The left panels give results for a $1.5 R_{J}$ companion. The right panels give results for a $1.0 R_{J}$ companion.

between the server and clients on the Beowulf cluster. A Beowoulf cluster belonging to the Korean Astronomy Observatory also provided computing resources for this calculation. C programming source code for either clientserver communication implementation is available upon request from the author.

The light solid line in Figure 8 shows the detection probability, $P_{\epsilon}\left(a, R_{p}\right)$, for three representative stars in order of increasing apparent magnitude from top to bottom and for the 2 companion radii, 1.0 and $1.5 R_{J}$, on the left and right, respectively. In general, the probability nears $100 \%$ completion for orbital periods $\lesssim 1.0$ day and then has a power law fall off toward longer orbital periods. The falloff in the detection probability toward longer orbital periods partially results from the requirement of observing more than one transit. The large drop in the detection probability around 0.5 and 1.0 day orbital periods results from the selection criteria we impose. The narrow, non-zero spikes in the detection probability near the 0.5 and 1.0 day orbital periods result from injecting a transit at this period, but the BLS method returns a best-fit period typically at the $\sim 0.66$ day alias.

Figure 8 shows the detection probability with 3.3 times higher resolution in orbital period and a lower, 1\%, error in the detection probability at fixed orbital period than the actual calculation. Thus, the figure resolves variability in the detection probability as a function of orbital period for probabilities $\gtrsim 1 \%$. However, such fine details have negligible impact on the results.

\subsection{Calculating $P_{T, i}\left(a, R_{p}\right)$}

The probability for a transit to occur is $P_{T}=\left(R_{\star}+\right.$ $\left.R_{p}\right) / a$. This transit probability assumes the transit is equally detectable for the entire possible range of orbital inclinations that geometrically result in a transit. As $\cos i$ for the orbit approaches $\left(R_{\star}+R_{p}\right) / a$ the transit length and depth decreases, degrading the transit $\mathrm{S} / \mathrm{N}$. We address this when computing $P_{\epsilon}$ by injecting the transit with an even distribution in $\cos i$ between the geometric limits for a transit to occur. Thus, $P_{T}$ represents the overall probability for a transit with high enough inclination to begin imparting a transit signal, while the detailed variation of the light curve signal for varying inclination takes place when calculating $P_{\epsilon} . P_{T}$ is shown as the dashed light line in Figure 8 The heavy solid line in Figure 8 is the product of $P_{\epsilon}$ and $P_{T}$.

\subsection{Calculating $P_{\text {mem }}$}

The Monte Carlo calculation requires knowledge of the stellar properties, and the given properties are only valid if the star is in fact a bona fide cluster member. An estimate of the field-star contamination from the CMD provides only a statistical estimate of the cluster membership probability. Based on the study of the mass function and field contamination in Burke et al. (2004), we estimate the cluster membership probability, $P_{\text {mem }}$, as a function of stellar mass. In brief, we start with a subsample of stars based on their proximity to the best-fit cluster isochrone (selection on $\chi_{\text {mem }}^{2}<0.04$, see \$5). This sample contains $N_{\star} \sim 2700$ potential cluster members, and the heavy points in Figure 5 mark this cluster sample in the CMD. The best-fit isochrone allows an estimate of the stellar mass for each member of the cluster sample, and we separate the sample into mass bins. Repeating this procedure on the outskirts of the observed field of view, scaled for the relative areas, provides an estimate of the field-star contamination in a given mass bin. We fit $P_{\text {mem }}$, given in discrete mass bins, with a smooth spline fit for interpolation.

The solid line in Figure 9 shows $P_{\text {mem }}$ as a function of stellar mass. The corresponding probability is given on the right side ordinate. The clear histogram shows the distribution of the potential cluster members as a function of mass. The lower shaded histogram shows the product of the potential cluster members histogram and $P_{\text {mem }}$. This results in effectively $N_{\star, \text { eff }} \sim 870$ cluster members in total. For reference, the corresponding apparent $I$-band magnitude is given along the top.

\section{RESULTS}

\subsection{Results Assuming a Power-law Orbital-period Distribution}

The previous section describes the procedure for calculating the sensitivity of the survey to detect planetary companions as a function of semimajor axis. The results from this calculation enable us to place an upper limit on the fraction of cluster members harboring closein companions given the null result. However, calculating the upper limit over a range of orbital periods necessitates assuming a distribution of orbital periods for the planetary companions. Radial velocity surveys characterize the distribution of extrasolar planets in period as $d n \propto P^{-\gamma} d P$, with $0.7 \lesssim \gamma \lesssim 1.0$, corresponding to $d n \propto a^{-\beta} d a$, with $0.5 \lesssim \beta \lesssim 1.0$ (Stepinski \& Black 2001; Tabachnik \& Tremaine 2002). These studies fit the entire range of orbital periods ranging from several days to several years. More recently, after an increase in 


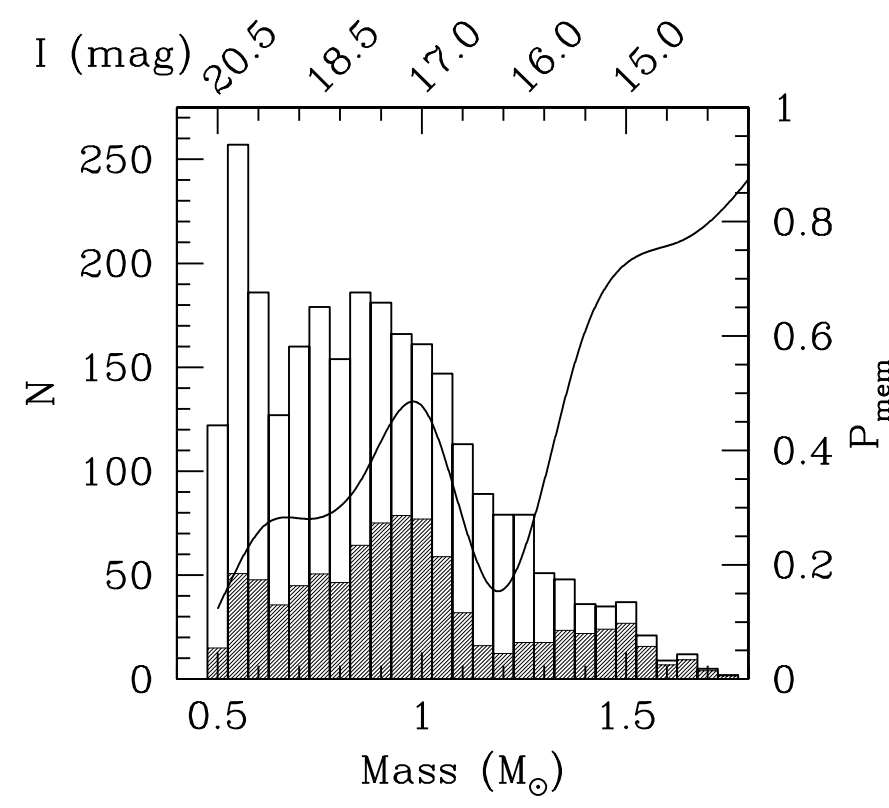

Fig. 9.- Distribution of the potential cluster members as a function of stellar mass (open histogram). The solid line shows the membership probability (right hand ordinate) as a function of stellar mass. The shaded histogram shows the product of the potential cluster member histogram and the cluster membership probability. The corresponding apparent $I$-band magnitude is given along the top.

the number of extrasolar planet discoveries, Udrv et al. (2003) confirm a shortage of planets with $10 \lesssim P \lesssim 100$ day orbits. Thus, the period distribution may take on different values of $\gamma$ in the $P \lesssim 10$ day and $P \gtrsim 100$ day regimes.

The initial extrasolar planet discoveries via the transit technique had periods less than 3.0 days (Konacki et al. 2004). The detection of these "Very Hot Jupiters," contrasted with the results from radial-velocity surveys, which demonstrated a clear paucity of planets with $P \lesssim$ 3.0 days. After accounting for the strong decrease in sensitivity of field transit surveys with increasing period, Gaudi et al. (2005) demonstrated the consistency between the apparent lack of VHJ companions in the radial velocity surveys and their discovery in transit surveys. They further demonstrated that VHJ appear to be intrinsically much rarer than HJ (with $3 \leq P /$ day $\leq 9$. We will therefore treat VHJ as HJ distinct populations.

Due to the incomplete knowledge of the actual period distribution of extrasolar planets and its possible dependence on the properties of the parent star, we provide upper limits assuming an even logarithmic distribution of semimajor axis. Thus, we assume a form of the joint probability distribution of the semimajor axis and $R_{p}$ given by

$$
\frac{d^{2} p}{d R_{p} d a}=k \delta\left(R_{p}-R_{p}^{\prime}\right) a^{-1},
$$

where $k$ is the normalization constant, $\delta$ is the Dirac delta function, and $R_{p}^{\prime}$ is the planet radius. We initially give

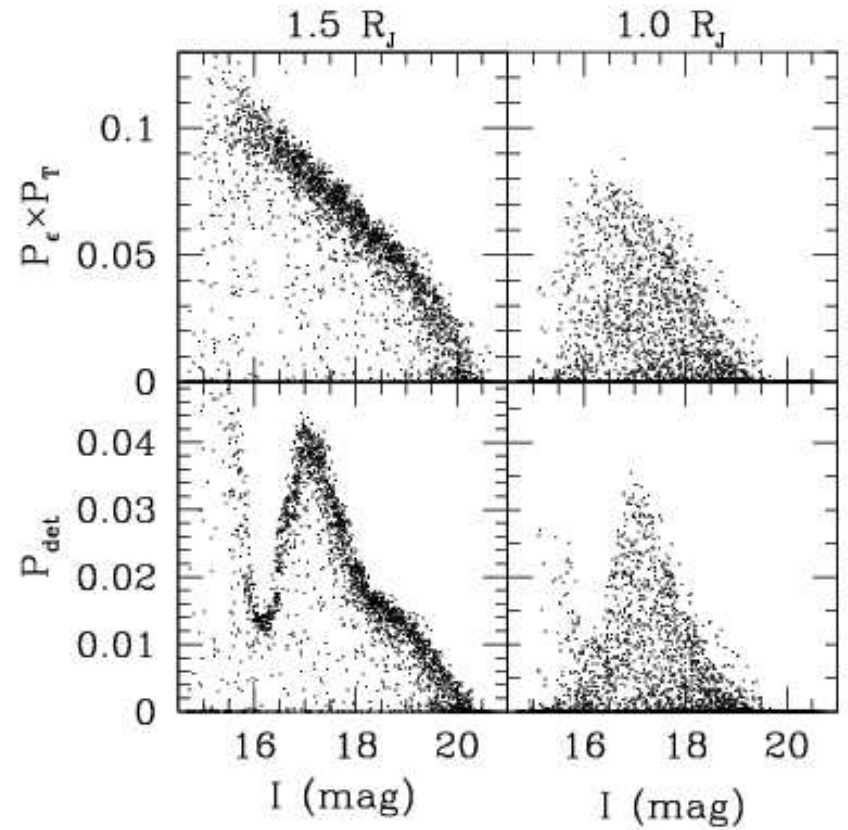

FIG. 10.- Probability for transit detection as a function of the apparent $I$-band magnitude assuming an even logarithmic distribution in semimajor axis from $1.0<P<3.0$ day. The top panels assume $P_{\mathrm{mem}}=1.0$. The left panels show results for a $1.5 R_{J}$ companion. The right panels show results for a $1.0 R_{J}$ companion. The bottom panels are the same as the top panels, but they take into account the membership probability $P_{\text {mem }}$.

results for $R_{p}^{\prime}=1.0$ and $1.5 R_{J}$. We follow Gaudi et al. (2005) and show results for HJ $(3.0<\mathrm{P}<9.0$ day $)$ and VHJ $(1.0<\mathrm{P}<3.0$ day) ranges. In addition, we show results for a more extreme population of companions with $P_{\text {Roche }}<P<1.0$ day, where $P_{\text {Roche }}$ is the orbital period at the Roche separation limit, which we designate as Extremely Hot Jupiter (EHJ). Assuming a negligible companion mass, the Roche period depends solely on the density of the companion. Jupiter, Uranus, and Neptune have nearly the same $P_{\text {Roche }} \sim 0.16$ day.

Figure [10 shows the probability for detecting a VHJ (1.0 day $\leq P \leq 3.0$ day) companion with an even logarithmic distribution in semimajor axis as a function of apparent $I$-band magnitude. The left and right panels show results for a 1.5 and $1.0 R_{J}$ companion, respectively. The top panels of Figure 10 show the probability for detecting an extrasolar planet, $P_{\mathrm{det}}$, assuming $P_{\mathrm{mem}}=1.0$. The bottom panels show $P_{\text {det }}$ after taking into account $P_{\text {mem }}$. The results for $1.0 R_{J}$ companions broadly scatter across the full range of detection probability. However, the $1.5 R_{J}$ companion results delineate a tight sequence in detection probability as a function of apparent magnitude.

The $1.5 R_{J}$ companion signal lies many times above the rms scatter in the light curve (see Figure 11). Thus, a single measurement contributes a large fraction of the $\mathrm{S} / \mathrm{N}$ required for detection. In this limit, the observing window function mainly determines the detection probability, and as we show in $\$ 9.2$ the result is similar to results obtained by the theoretical detection probability framework of Gaudi (2000). However, the $1.0 R_{J}$ companion transit signal comes closer to the detection 


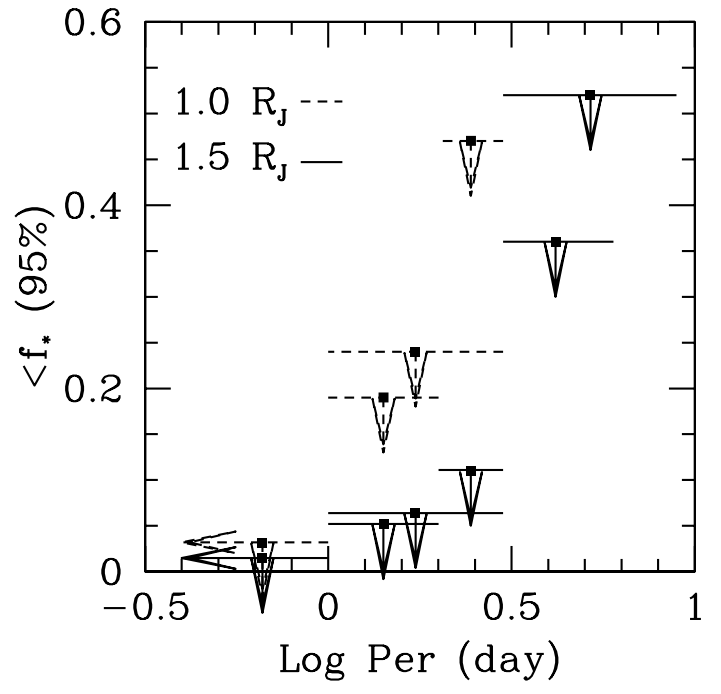

FIG. 11.- Upper limit (95\% Confidence) on the fraction of stars in the cluster with companions for several ranges in orbital period assuming an even logarithmic distribution in semimajor axis. The solid lines show results for a $1.5 R_{J}$ companion. The dash lines show results for a $1.0 R_{J}$ companion.

threshold. Pepper \& Gaudi (2005) describe the sensitivity of a transit survey as a function of planet radius. The sensitivity of a transit survey depends weakly on $R_{p}$ until a critical radius is reached when the $\mathrm{S} / \mathrm{N}$ of the transit falls rapidly. The sensitivity of the survey for $1.0 R_{J}$ is near this threshold, hence the large scatter in the detection probability.

With the detection probabilities for all stars in the survey for the assumed semimajor axis distribution, we can calculate the expected number of detections scaled by the fraction of cluster members with planets. Thus, from the Poisson distribution, a null result is inconsistent at the $\sim 95 \%$ level when $N_{\text {det }} \sim 3$. This allows us to solve for the $95 \%$ confidence upper limit on the fraction of cluster members with planets using Eq. 3. This gives,

$$
f_{\star} \leq 3.0 / \sum_{i=1}^{N_{\star}} P_{\text {det }, \mathrm{i}} \quad(95 \% \text { c.l. }) .
$$

Figure 11 shows the $95 \%$ confidence upper limit on the fraction of stars with planets in NGC 1245 for several ranges of orbital period. The solid and dashed lines give results for 1.5 and $1.0 R_{J}$ companions, respectively. For $1.5 R_{J}$ companions we limit the fraction of cluster members with companions to $<1.5 \%,<6.4 \%$, and $<52 \%$ for EHJ, VHJ, and HJ companions, respectively. For 1.0 $R_{J}$ companions, we find $<2.3 \%$ and $<15 \%$ have EHJ and VHJ companions, respectively.

The detection probability decreases rapidly with orbital period beyond 1.0 day. As a result, the survey does not reach the sensitivity needed to place an interesting upper limit on $1.0 R_{J}$ companions beyond $P>3.0$ day.

We further divide the VHJ period range and show upper limits for the period ranges $1.0 \nmid P /$ day $i 2.0$ and $2.0<P /$ day $<3.0$, which we denote as $P_{12}$ and $P_{23}$. For $1.5 R_{J}$ companions we limit $f_{\star}$ to $<5.2 \%$ and $<11 \%$ for $P_{12}$ and $P_{23}$, respectively. For $1.0 R_{J}$ companions we limit $f_{\star}$ to $<19 \%$ and $<47 \%$ for $P_{12}$ and $P_{23}$, respec-

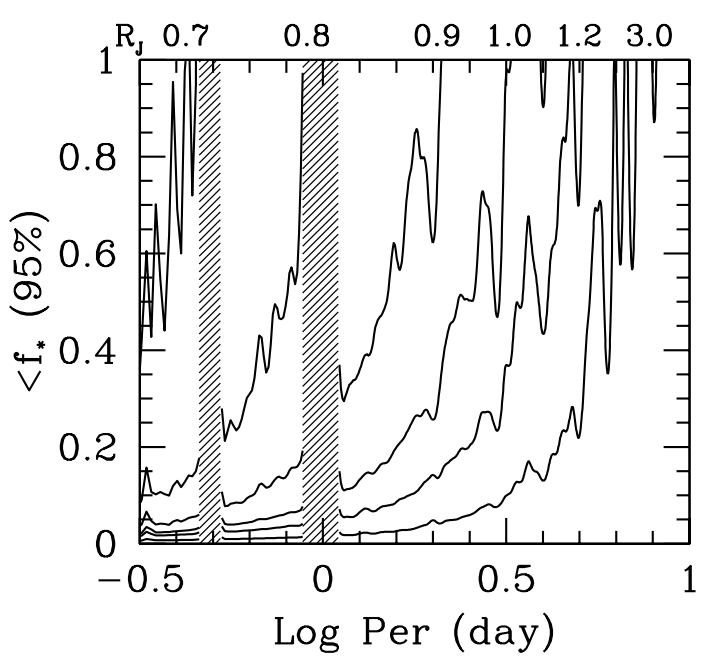

FIG. 12.- Upper limit (95\% Confidence) on the fraction of stars in the cluster with companions for several companion radii as label along the top. The result for a $1.0 R_{J}$ companion is based on the entire sample, whereas the results for the other companion radii are based on a subsample of $N_{\star}=100$ stars. The shaded regions denote orbital periods removed by the selection criteria in order to eliminate false-positive transit detections that occur around the diurnal period and 0.5 day alias.

tively. We also divide the HJ period range and limit $f_{\star}$ for $1.5 R_{J}$ companions in the $3.0<P /$ day $<6.0$ to $<36 \%$.

\subsection{Results for Other Companion Radii}

Due to computing limitations we calculate detection probabilities for the entire cluster sample only for 1.5 and $1.0 R_{J}$ companions. In $\$ 8$ we show that an upper limit determination using a subsample of the stars with size $N_{\star, \mathrm{SS}}=100$ approximates the results based on the entire stellar sample. Thus, we calculate upper limits for a variety of companion radii using $N_{\star, \text { SS }}=100$ randomly chosen stars in the sample. Instead of showing upper limit results over a range of orbital periods, we derive upper limits at fixed period by replacing the semimajor axis distribution with a $\delta\left(a-a_{o}\right)$ function in Equation 5. To obtain results at fixed period, each star has a different $a_{o}$ that depends on the stellar mass. Figure 12 shows the upper limit on the fraction of stars with planets in the survey as a function of orbital period. The lines show results for various values of the companion radius in terms of $R_{J}$ as indicated by the label next to each line along the top of the figure. The shaded regions denote orbital periods removed by the selection criteria in order to eliminate false-positive transit detections that occur around the diurnal period and 0.5 day alias. At smaller companion radii, the transit $\mathrm{S} / \mathrm{N} \propto R_{p}^{2}$ drops quickly. Toward larger companion radii the $\mathrm{S} / \mathrm{N}$ of the transit saturates and the observational window function increasingly dominates the survey effectiveness. The survey cannot detect companions with $R_{p}>3.5 R_{J}$ as the transit/eclipse becomes too deep given the removal of measurements that deviate by more than $0.5 \mathrm{mag}$ from the mean light-curve level.

\section{ERROR IN THE UPPER LIMIT}


In this section we discuss several sources of error present when determining an upper limit on the fraction of stars with planets.

\subsection{Error When Using a Subsample}

Computing power limitations discourage calculating detection probabilities over the entire cluster sample. Thus, we first characterize the error associated with determining an upper limit using only a subset of the entire cluster sample. Starting with Equation 6] we derive an error estimate when using a subsample by the following means. Replacing the summation over $P_{i \text {,det }}$ with the arithmetic mean, $\left\langle P_{\text {det }}\right\rangle$, Equation [6] becomes

$$
f_{\star}=3.0 /\left(N_{\star}\left\langle P_{\text {det }}\right\rangle\right) .
$$

By propagation of errors, the error in the upper limit is given by

$$
\sigma_{f}=\frac{3.0}{N_{\star}} \frac{\sigma_{\langle P\rangle}}{\left\langle P_{\text {det }}\right\rangle^{2}},
$$

where $\sigma_{\langle P\rangle}$ is the error in the mean detection probability. The error in the mean detection probability scales as $\sigma_{\langle P\rangle}=\sigma_{P} / \sqrt{N_{\star, S S}}$, where $\sigma_{P}$ is the intrinsic standard deviation of the distribution of $P_{i \text {,det }}$ values and $N_{\star, \text { SS }}$ is the size of the subsample.

We empirically test this error estimate by calculating the upper limit with subsamples of increasing size. The small points in Figure 13 show the upper limit on the fraction of stars with planets as a function of the subsample size. The upper limit calculation assumes an even logarithmic distribution of semimajor axis for companions with $1.0 \leq P \leq 3.0$ day for 1.5 and $1.0 R_{J}$ radius companions, top and bottom panels, respectively. Neighboring columns of upper limits differ by a factor of 2 in the subsample size. We randomly draw stars from the full sample without replacement, making each upper limit at fixed sample size independent of the others. The dashed line represents the upper limit based on the full cluster sample.

The distribution of upper limits around the actual value possesses a significant tail toward higher values. This tail results from the significant number of stars with $P_{\text {tot }}=0.0$. At fixed sample size, the large square point represents the mean upper limit. Using subsamples sizes of $N_{\star, \text { SS }} \lesssim 20$ tends to systematically overestimates the true upper limit. The open star symbol represents the $1-\sigma$ standard deviation of the distribution at fixed sample size. The solid line shows the error estimate from Equation 8 Despite the non-Gaussian nature of the underlying distribution, the error estimate in the upper limit roughly corresponds with its empirical determination especially toward increasing $N_{\star, \text { SS }}$ where the systematic effects become negligible. From Figure [13 we conclude that adopting $N_{\star, S S} \gtrsim 100$ provides adequate control of the random and systematic errors in calculating an upper limit, without becoming numerically prohibitive. This verifies the procedure for estimating the upper limit for a variety of companion radii in $\$ 7.2$

\subsection{Error in Determining Sample Size}

Up to this point, we have mainly addressed sources of error directly associated with determining $P_{\epsilon}$. However, the upper limit error budget contains an additional source of error from uncertainties in determining

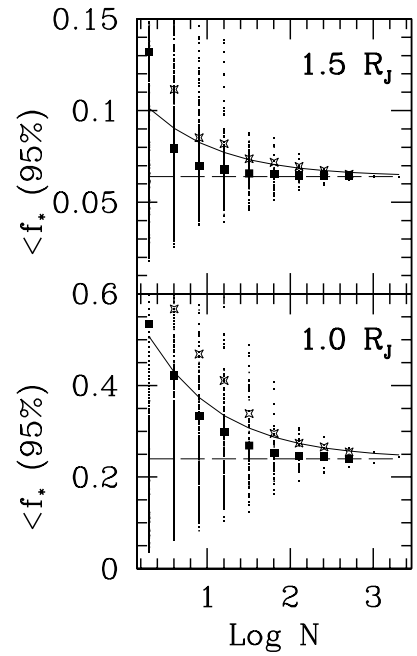

FIG. 13. - Estimates for the upper limit (95\% Confidence) on the fraction of stars in the cluster as a function of the sample size employed in making the estimate are shown as small points. We have assumed an even logarithmic distribution in periods between $1.0<P /$ day $<3.0$ orbital period. The dash line shows the upper limit based on the entire sample. The average upper limit at fixed sample size is given by square points. The sequence of open stars gives the standard deviation in the distribution of upper limits at fixed sample size. The solid line shows the error model estimate for the standard deviation in the upper limit. The top panel gives results for a $1.5 R_{J}$ companion, and the bottom panel gives results for a $1.0 R_{J}$ companion.

$P_{\text {mem. }}$. This additional source of error directly relates to the accuracy in determining the number of single mainsequence stars in the survey.

We characterize this error as follows. At fixed orbital period, $\left\langle P_{\text {det }}\right\rangle=\left\langle P_{\text {mem }} P_{\epsilon} P_{T}\right\rangle$. Given that $P_{\text {mem }}$ is nearly independent of the other terms, the previous average is separable, such that $\left\langle P_{\text {det }}\right\rangle=\left\langle P_{\text {mem }}\right\rangle\left\langle P_{\epsilon} P_{T}\right\rangle$. This separation changes the derived upper limit by a negligible $0.3 \%$ relative error. The separation allows us to rewrite Equation 7 as

$$
f_{<, 95}=3.0 /\left(N_{\star, \text { eff }}\left\langle P_{\epsilon} P_{T}\right\rangle\right),
$$

where $N_{\star, \text { eff }}=N_{\star}\left\langle P_{\text {mem }}\right\rangle$ is the effective number of cluster members in the sample after taking into account background contamination. Thus, $N_{\star, \text { eff }}$ carries equal weight with $\left\langle P_{\epsilon} P_{T}\right\rangle$ in the upper-limit error budget.

The ability to determine $N_{\star, \text { eff }}$ accurately provides an advantage for transit surveys toward a rich stellar cluster rather than toward a random galactic field. Even though methods based on the cluster CMD statistically determine cluster membership, they concentrate on a narrow main-sequence region to search for planets where the cluster counts significantly outweigh the background contamination counts. By concentrating on the main sequence of a cluster, this survey has only $\sim 68 \%$ contamination by background stars. In contrast, random galaxy fields contain $\gtrsim 90 \%$ contamination by subgiant and giant stars for $\mathrm{V}<11$ surveys (Gould \& Morgan 2003). Overall, $N_{\star, \text { eff }}$ has an $8 \%$ error, which propagates to a relative error of $8 \%$ in the upper limit. The error in $N_{\star, \text { eff }}$ comes from subtracting the star counts observed within a $12.7^{\prime}$ radius of the cluster center by the control field star counts outside this radius. The error is larger 
than the Poisson error of $N_{\star, \text { eff }}=870$ since the control field star count is scaled to match the larger cluster field area.

\subsection{Error Due to Blends and Binaries}

The final source of error we address results from stellar blends due to physical binaries or chance, line-of-sight associations. The additional light from an unresolved blend dilutes a transit signal from one component of the blend. Thus, we overestimate the ability to detect a transit around blends. However, a compensatory effect arises since the extra light from a blend results in an overestimate in the stellar mass and radius, which in turn results in modeling a shallower transit. Modeling such details is not possible without knowing the binary nature for each object, but we can estimate the number of stars affected by assuming binary star statistics as measured in the field. Due to low stellar crowding, we estimate chance blends have a negligible effect in comparison to physically associated binaries (Kiss \& Bedding 2005). Finding charts in Figure 6] demonstrate the stellar crowding conditions of the survey.

The latest Coravel radial velocity survey dedicated to F7-K field dwarfs (Halbwachs et al. 2004) and the visual binary and common proper motion pairs survey of Eggenberger et al. (2004) provide the basis for the binary star estimates. Overall they find a binary frequency of $56 \%$ for systems with $\log (P /$ day $) \leq 6.31$. However, due to the strong dependence of luminosity on the stellar mass only systems with mass ratio, $q>0.6$, significantly contribute light to dilute the transit signal. For lower mass ratios the lower mass component contributes $<20 \%$ of the total system flux. When taking binaries across the entire range of orbital periods the mass-ratio distribution peaks near $q \sim 0.2$ and slowly drops toward higher q (Duquennov \& Mavor 1991). From Figure 10 in Duquennov \& Mavor (1991), only $\sim 20 \%$ of their binary systems have $q>0.6$. Thus, if the binary statistics for the cluster matches the field dwarfs, transit dilution occurs for $\sim 11 \%$ of the stellar sample. The radial velocity survey for binaries in the Pleiades and Praesepe clusters reveals consistency with the frequency of binaries in the field surveys (Halbwachs et al. 2004).

In principle, the data from this survey can also answer whether the binary statistics of the cluster matches the field dwarfs. However, the statistical methods and selection criteria described in this study do not optimally detect interacting and eclipsing binaries. Additionally, in order to reach planetary companion sensitivities, we remove light-curve deviations beyond $0.5 \mathrm{mag}$ as discrepant, which removes the deep eclipses.

\subsection{Overall Error}

The errors involved with determining the number of cluster members dominates the error budget in determining the upper limit. However, as discussed in $\$ 6$ this is only true if one quantifies and corrects for the systematic overestimate in detection probability due to a reduction in the transit signal from the procedures of generating and correcting the light curve. For instance, at the median stellar brightness for this survey, the detection probability is overestimated by $>15 \%$ for orbital periods $>4.0$ day and $>1.0$ day for 1.5 and $1.0 R_{J}$ companions, respectively, without correction. Since we char- acterize this systematic effect, the error in determining the number of cluster members dominates the error budget.

Additionally, the potential for a large contamination of binaries diluting the transit signal necessitates an asymmetrical error bar. We roughly quantify the error estimate resulting from binary contamination from the field dwarf binary statistics. From the arguments in the previous section, we adopt $11 \%$ as a $1-\sigma$ systematic fractional error due to binary star contamination. Overall, combining this systematic error with the $7 \%$ fractional error in determining the cluster membership, upper limits derived from the full stellar sample contain a ${ }_{-7 \%}^{+13 \%}$ fractional error.

\section{DISCUSSION}

Along with this work, several other transit surveys have quantified their detection probability from actual observations in an attempt to constrain the fraction of stars with planets or quantify the consistency with the solar neighborhood radial velocity planet discoveries (Gilliland et al. 2000; Weldrake et al. 2005; Mocheiska et al. 2005; Hidas et al. 2005; Hood et al. 2005). Unfortunately, a direct comparison of upper limits from this work with these other transit surveys cannot be made. Until this study, none of the previous studies have quantified the random or systematic errors present in their techniques in sufficient detail to warrant a comparison. Additionally, previous studies do not have quantifiable selection criteria that completely eliminate falsepositive transit detections due to systematic errors in the light curve, a necessary component of an automated Monte Carlo calculation.

\subsection{Initial Expectations vs. Actual Results}

In the meantime, we can discuss why the initial estimate of finding two planets assuming $1 \%$ of stars have $R_{J}$ companions evenly distributed logarithmically between 0.03 to $0.3 \mathrm{AU}$ (Burke et al. 2003) compares to the results from this study, which indicate that we expected to detect 0.1 planets. The initial estimates for the detection rate are based on the theoretical framework of Gaudi (2000). Given a photometric noise model, observational window, and $\mathrm{S} / \mathrm{N}$ of the transit selection criteria, the theoretical framework yields an estimate of the survey detection probability. This theoretical detection probability coupled with a luminosity function for the cluster determines the expected number of detections. As we show next, the initial estimates did not account for the light curve noise floor or detector saturation, and contain optimistic estimates for the sky background and luminosity function. In addition, the initial estimates could not have accounted for the $50 \%$ reduction in signal for the majority of the light curves due to the detector error discussed in 3.4 Finally, as discussed in detail by Pepper \& Gaudi (2005) and demonstrated explicitly here, the detection probability is very sensitive to the precise error properties near the critical threshold of detection, which for this survey is just reached for $R_{J}$ companions.

The top panels of Figure 14 compare the detection probability of the Monte Carlo calculation of this study to the initial theoretical estimate. The small points replicate the Monte Carlo results from the top panels of Fig- 


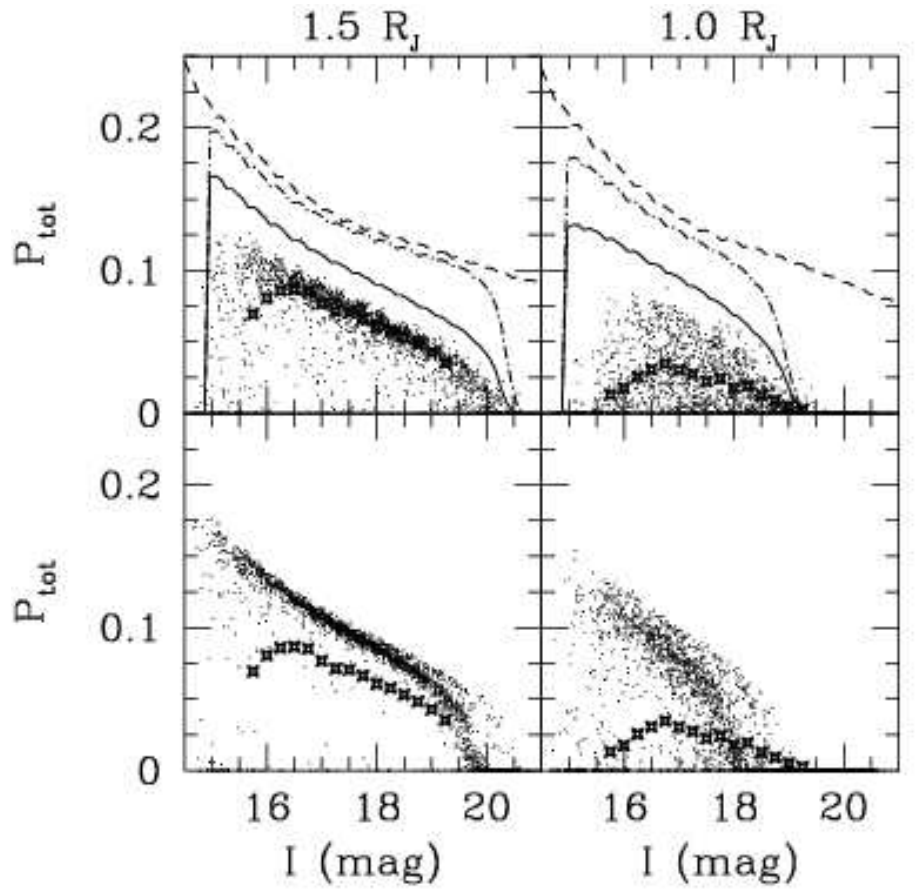

FIG. 14.- Top: Probability for transit detection as a function of the apparent $I$-band magnitude assuming an even logarithmic distribution in semimajor axis from $1.0<P<3.0$ day and $P_{\text {mem }}=1.0$ using the Monte Carlo calculation of this study(small points). The binned average of the Monte Carlo results is denoted by open stars. The dash line shows the expected probability for transit detection based on a theoretical calculation prior to this survey. The dot dash line shows the theoretical probability for transit detection assuming a photometric noise model appropriate for the survey. The solid line shows the theoretical probability for transit detection with an accurate photometric noise model for the survey and including the effects of limb darkening. The left panel shows $1.5 R_{J}$ companion results. The right panel shows $1.0 R_{J}$ companion results. Bottom: Shows the theoretical probability for transit detection allowing each star of the survey to have its empirically determined photometric noise and including the effects of limb darkening (small points). The open stars are reproduced from the top panels.

ure 10 while the dashed line shows the detection probability based on the initial theoretical expectations. The initial theoretical expectations clearly overestimate the detection probability. The bright end continues to rise due to ignoring the effects of detector saturation and the photometric noise floor. The faint end does not cutoff due to an underestimated sky brightness. The initial estimate of the sky brightness, $19.5 \mathrm{mag} \operatorname{arcsec}^{-2}$, compares optimistically to the range of sky brightnesses encountered during the actual observations. The sky varied between 17.5 and $19.0 \mathrm{mag} \operatorname{arcsec}^{-2}$ over the course of the observations. The full lunar phase took place near the middle of the observation, and the Moon came within $40^{\circ}$ of the cluster when nearly full.

The initial estimate for the cluster luminosity function simply selected cluster members via tracing by eye lines that bracket the main sequence in the CMD. This crude technique led to an estimated 3200 cluster members down to $I \sim 20$. A careful accounting of the field star contamination results in only $\sim 870$ cluster members in the survey. The luminosity function overestimate and the expected sensitivity to transits around the bright and faint cluster members leads to a factor of 4-5 overestimate in the number of cluster members in the survey. Additionally, the factor of 4-5 overestimate of the initial detection probability when compared to binned average detection probability for the Monte Carlo results (open stars in Figure 14), easily accounts for the factor of 20 difference in the overall number of expected detections (for $R=R_{J}$ ).

\subsection{Improving Theoretical Expectations}

Clearly, accurate and realistic transit detection statistics requires more detailed analysis than these early estimates and more careful theoretical work has already been done (Pepper \& Gaudi 2005). In the case of an open cluster, delineating cluster membership by tracing the main sequence in the CMD overestimates the number of cluster members. A careful subtraction of the field contamination is necessary in order to extract an accurate cluster-member count.

A photometric noise model that accurately reflects the quality of observations is the next step in correctly calculating a theoretical detection probability. From Figure 1 we estimate the actual photometric noise present in the data. This includes the proper sky measurement and systematic floor in the photometric precision. With a noise model similar to the lower solid line in Figure 1] we recalculate the theoretical detection probability. The dot dash line in Figure 14 shows the resulting detection probability still overestimates the Monte Carlo results. However, it does agree with the faint-end cutoff of the Monte Carlo calculation. We impose the bright-end cutoff due to saturation effects at the same magnitude as the observed increase in light curve rms as shown in Figure 1

For these results we include an additional effect not taken into account by Gaudi (2000). We multiply the transit $\mathrm{S} / \mathrm{N}$ selection criteria, Equation 5 of Gaudi (2000), by $\sqrt{\max \left(N_{\text {obs }}, 1.7\right)}$, where $N_{\text {obs }}$ is the typical number of transits detected throughout the observing run. The $N_{\text {obs }}=1.7$ floor in this factor corresponds to the requirement of observing the transit twice multiplied by the observing efficiency. For simplicity, we take $N_{\text {obs }}=N_{\text {tot }} / P \times 0.2$, where $N_{\text {tot }}=16$, the length of the observing run in days, and the factor of 0.2 accounts for the actual observational coverage encountered during the run.

Given that the theoretical calculation still overestimates the Monte Carlo results, to increase the realism of the theoretical detection probability, we include a linear limb-darkening law, which effectively weakens the transit depth. We solve for the factor $G$, Equation 6 of Gaudi (2000), assuming a linear limb-darkening parameter, $\mu=0.6$, for all stars. The inclusion of limb darkening significantly impacts the theoretical detection probability as the solid line in Figure 14 demonstrates. Although the theoretical detection probability still overestimates the upper envelope of results from the Monte Carlo calculation, the level of agreement, after including an accurate photometric noise model and limb darkening, shows significant improvement over the initial estimates.

Despite the improved agreement, the Monte Carlo detection probability calculation shows significant scatter at fixed magnitude. The theoretical probability treats all stars at fixed magnitude as having the same noise properties. With the theoretical detection probability we 
can address whether the scatter in detection probability at fixed magnitudes results from the observed scatter in noise properties at fixed magnitude as shown in Figure 1 Thus, we calculate a theoretical detection probability for each star individually using the measured rms in the light curve for each star to determine the theoretical transit $\mathrm{S} / \mathrm{N}$ selection criteria using Equation 5 of Gaudi (2000). The small points in the bottom panels of Figure 14 show the resulting theoretical detection probability.

Some of the scatter in detection probability results from the scatter in noise properties as a function of magnitude. The heavy star points represent the average Monte Carlo detection probability in 0.25 magnitude bins. In the case of the $1.5 R_{J}$ companions, the signal is large in comparison to the photometric noise. The left panels of Figure 14] demonstrate the theoretical detection probability overestimates the Monte Carlo detection probability by only $20 \%$. However, the closer the transit signal approaches the systematic and rms noise, the theoretical detection probability strongly overestimates the actual detection probability. In the case of $1.0 R_{J}$ companions (right panels of Figure 14), the theoretical calculation overestimates the Monte Carlo results by $80 \%$. Thus, we urge caution when relying on a theoretical detection probability when the survey is near the critical threshold for transit detection. Such is the case for 1.0 $R_{J}$ companions in this survey.

\subsection{Planning Future Surveys}

Even though the theoretical calculation overestimates the absolute detection probability by a factor of $<2$, tests on a small sample of stars with the Monte Carlo calculation reveal it provides a much higher relative accuracy. Thus, the computationally efficient theoretical calculation allows us to examine the relative change in the detection probability for a given change in survey parameters. For planning future surveys it is essential to decide between increasing the number of stars by observing another cluster or improving the detection probability by increasing the length of observations on a single cluster. As shown in 77 the upper limit scales linearly with the sample size, thus keeping everything else constant, increasing the sample size by a factor of 2 improves the upper limit by a factor of 2 .

Using the theoretical detection probability framework, we can quantify the improvement in sensitivity for a survey twice as long. We assume a survey twice as long consists of an observing window identical to the current survey for the first half and repeats the observing window of the current survey for the latter half. The upper limit improves only by a factor of 1.3 for a logarithmic distribution of VHJ planets. However, the upper limits for HJs with 3.0 to 9.0 day orbital periods decrease by a factor of 2.6. Thus, not only is it more efficient to observe this cluster twice as long, but the analysis of Gaudi et al. (2005) reveals a 5-10 times larger HJ population than the the VHJ population. This strongly suggests transit surveys with a single observing site require month long runs for maximum efficiency in detecting HJ companions.

Figure 14 reveals little improvement in the detection probability occurs for increasing the photometric precision, at least for $1.5 R_{J}$ companions. To first order, the photometric precision determines the faint-end cutoff in the detection probability. Thus, a lower sky back- ground or improved photometric precision predominately effects the number of stars in the survey rather than the detection probability. However, improving the photometric precision does lead to increasing the sensitivity for smaller radius companions. In the case of 1.0 $R_{J}$ companions, the rms in the light curve typically is $\lesssim 1.8$ times lower than the transit signal. As shown in the previous section, the theoretical detection probability breaks down for such low precision. In the case of $1.5 R_{J}$ companions, the rms in the light curve typically is $\lesssim 4$ times lower than the transit signal. Thus, for the $1.0 R_{J}$ results to reach the same sensitivity as the 1.5 $R_{J}$ results, improvement in the light curve rms is necessary until the transit $\mathrm{S} / \mathrm{N}$ is above a critical threshold when the detection probability is weakly dependent on $R_{p}$ (Pepper \& Gaudi 2005).

According to a recent review of radial velocity detected planets, $1.2 \pm 0.3 \%$ of solar neighborhood stars have HJ companions (Marcy et al. 2005). This survey of NGC 1245 reached an upper limit of $52 \%$ of the stars having $1.5 R_{J}$ HJ companions. As mentioned previously, a survey lasting twice as long can reduce this upper limit to $21 \%$. Reaching similar sensitivity as the radial velocity results requires observing additional clusters in order to increase the number of stars in the sample. This survey has $\sim 870$ cluster members and $\sim 740$ of them have nonzero detection probability for $1.5 R_{J}$ VHJ companions. Hence a total sample size of $\sim 7400$ dwarf stars observed for a month will be needed to help constrain the fraction of stars with planets to a $2 \%$ level (comparable to radial velocity results). Assuming that the observed HJ frequency of $\sim 1 \%$ remains valid for a variety of stellar environments, we expect to detect one planet every 5000 dwarf stars observed for a month. Results for 1.0 $R_{J}$ companions without substantial improvement in the photometric precision likely will require a small factor larger sample size.

\section{CONCLUSION}

In this study we complete the analysis of a 19-night search for transiting extrasolar planets orbiting members of the open cluster NGC 1245. An automated transit search algorithm with quantitative selection criteria finds six transit candidates; none are bona fide planetary transits. Thus, this work also details the procedure for analyzing the null-result transit search in order to determine an upper limit on the fraction of stars in the cluster harboring close-in $R_{J}$ companions. In addition, we outline a new differential photometry technique that reduces the level of systematic errors in the light curve.

A reliable upper limit requires quantifiable transit selection criteria that do not rely on visual, qualitative judgments of the significance of a transit. Thus, we develop completely quantitative selection criteria that enable us to calculate the detection probability of the survey via Monte Carlo techniques. We inject realistic limb-darkened transits in the light curves and attempt their recovery. For each star we inject 100,000 transits at a variety of semimajor axes, orbital inclination angles, and transit phases, to fully map the detection probability for 2700 light curves consistent with cluster membership based on their position in the CMD. After characterizing the field contamination, we conclude the sample contains $\sim 870$ cluster members. 
When calculating a 95\% confidence upper limit on the fraction of stars with planets, we assume companions have an even logarithmic distribution in semimajor axis over several ranges of orbital period. We adopt the period ranges as outlined by Gaudi et al. (2005), for HJ and VHJ companions, and an as of yet undetected population with $\mathrm{P}<1.0$ day, which we denote as Extremely Hot Jupiters (EHJ). For NGC 1245, we limit the fraction of cluster members with $1.0 R_{J}$ companions to $<3.2 \%$ and $<24 \%$ for EHJ and VHJ companions, respectively. We do not reach the sensitivity to place any meaningful constraints on $1.0 R_{J}$ HJ companions. For $1.5 R_{J}$ companions we limit the fraction of cluster members with companions to $<1.5 \%,<6.4 \%$, and $<52 \%$ for EHJ, VHJ, and HJ companions, respectively.

We also fully characterize the errors associated with calculating the upper limit. We find the overall error budget separates into two equal contributions from error in the total number of single dwarf cluster members in the sample and the error in the detection probability. After correcting the detection probability for systematic overestimates that become increasingly important for detecting transits toward longer orbital periods (see \$6), we conclude that random and systematic errors in determining the number single dwarf stars in the sample dominate the error budget. \$d details the error analysis, and overall, we assign a ${ }_{-7 \%}^{+13 \%}$ fractional error in the upper limits.

In planning future transit surveys, we demonstrate that observing NGC 1245 for twice as long will reduce the upper limits for the important HJ period range more efficiently than observing an additional cluster of similar richness as NGC 1245 for the same length of time as this data set. To reach a $\sim 2 \%$ upper limit on the fraction of stars with $1.5 R_{J}$ HJ companions, where radial velocity surveys currently measure $1.3 \%$ (Marcy et al. 2005), we conclude a total sample size of $\sim 7400$ dwarf stars observed for a month will be needed. If $1 \%$ of stars have 1.5 $R_{J}$ HJ extrasolar planets, we expect to detect one planet every 5000 dwarf stars observed for a month. Results for $1.0 R_{J}$ companions without substantial improvement in the photometric precision likely will require a small factor larger sample size.

This publication was not possible in a timely manner without the gracious donation of computing resources by the following individuals: D. An, N. Andronov, M. Bentz, E. Capriotti, J. Chaname, G. Chen, X. Dai, F.
Delahaye, K. Denney, M. Dietrich, S. Dong, S. Dorsher, J. Escude, D. Fields, S. Frank, H. Ghosh, O. Gnedin, A. Gould, D. Grupe, J. Guangfei, C. Onken, J. Marshall, S. Mathur, C. Morgan, N. Morgan, S. Nahar, J. Pepper, B. Peterson, J. Pizagno, S. Poindexter, J. Prieto, B. Ryden, A. Steed, D. Terndrup, J. Tinker, D. Weinberg, R. Williams, B. Wing, J. Yoo. We thank C. Han for the donation of supercomputing resources belonging to the Korea Astronomy Observatory and Astrophysical Research Center for the Structure and Evolution of the Cosmos (ARCSEC) of Korea Science and Engineering Foundation (KOSEF) through Science Research Program (SRC) program. This publication makes use of supercomputer resources through the Cluster Ohio Project Rev3, an initiative of the Ohio Supercomputer Center, the Ohio Board of Regents, and the OSC Statewide Users

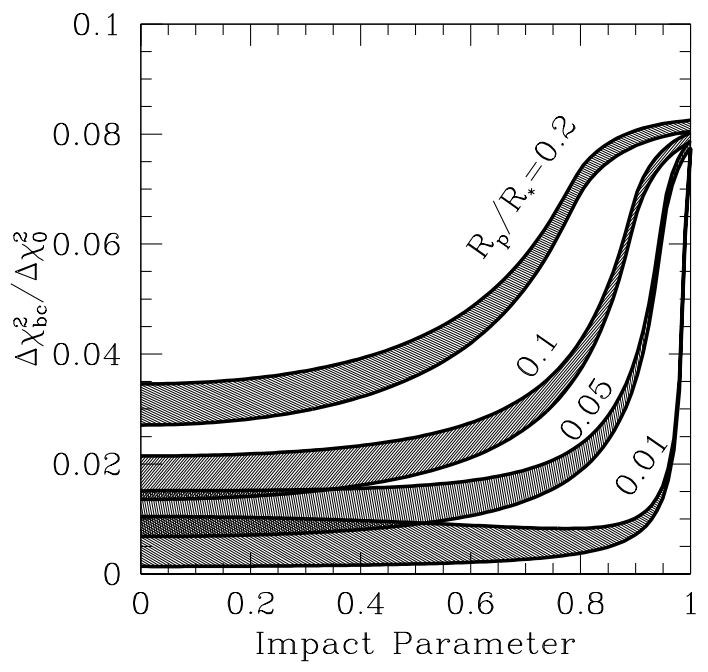

FIG. 15. - The lines show $\Delta \chi_{b c}^{2}$, the difference in $\chi^{2}$ between a boxcar fit to a planetary transit across a limb-darkened star and the exact model fit, normalized by $\Delta \chi_{0}^{2}$ the difference in $\chi^{2}$ between the exact model fit and a constant flux fix to the light curve. Each band is for a different planet/star radius ratio $R_{p} / R_{*}$, and the width of the band shows the variation in $\Delta \chi_{b c}^{2} / \Delta \chi_{0}^{2}$ for range of linear limb-darkening parameters $u_{1}=0.0-0.4$.

Group. This work was supported by NASA grant NAG513129 and a Menzel Fellowship from the Harvard College Observatory.

\section{APPENDIX}

In this appendix, we derive the boxcar fitting algorithm used to search for planetary transits. The original developers of this algorithm (Kovács et al. 2002), hereafter BLS, study its properties in the presence of Gaussian noise. We rederive the BLS method here in terms of the familiar language of $\chi^{2}$ minimization, demonstrating the equivalence of the two approaches (see also Aigrain \& Irwin 2004). In the process, we elucidate the meaning of some of the detection statistics introduced in Kovács et al. (2002). Finally, we quantify the accuracy of the boxcar approximation to transit light curves and discuss appropriate detection thresholds in the presence of correlated systematic errors as well as pure noise.

We begin by considering a series of flux measurements, $F_{k}$, taken at times $t_{k}$ with errors $\sigma_{F, k}$. The equation, $\bar{F} \equiv\left(\sum_{k} F_{k} / \sigma_{F, k}^{2}\right) /\left(\sum_{k} 1 / \sigma_{F, k}^{2}\right)$, defines the mean error-weighted flux of the measurements. We can write the fractional deviations from the mean as $m_{k} \equiv F_{k} / \bar{F}-1$ and the fractional errors as $\sigma_{k}=\sigma_{F, k} / \bar{F}$. For small deviations, the difference from the mean in magnitudes approximates $-1.086 m_{k}$.

A light curve of a star with transiting planet can be written as $F(t)=F_{0}[1-\delta(t)]$, where $F_{0}$ is the unobscured flux of the star, and $\delta(t)$ is the fractional drop in flux due to the transiting planet as a function of time. This generally 
depends on five parameters, namely the planet period $P$, the phase of the planet orbit $\phi$, the time it takes for the planet to cross one stellar radius, the ratio of the planet radius to stellar radius $\rho$, and the limb-darkening parameter of the star. We can write the fractional flux difference as $m(t)=F(t) / \bar{F}-1=m_{0}-\delta(t)-m_{0} \delta(t)$, where we have defined $m_{0}=F_{0} / \bar{F}-1$. We will assume that $\delta \ll 1$ and $m_{0} \ll 1$, and therefore $m(t) \simeq m_{0}-\delta(t)$.

For $\rho \ll 1$, and no limb darkening, the light curve $m(t)$ phased to the period $P$ reduces to a simple boxcar, $\delta=\rho^{2}$ when the planet is occulting the star and $\delta=0$ otherwise, with five parameters: the unocculted flux $m_{0}$, the transit period $P$, phase $\phi$, duration $\Delta t$, and depth $\delta$. This simple form allows an analytical solution for two of these parameters. For finite $\rho$ and limb darkening, the expression for $\delta(t)$ increases in complexity, but, as we show later, a simple boxcar still approximates the variability.

The likelihood $\mathcal{L}$ that the model $m(t)$ with parameters $\alpha=\left(m_{0}, \delta, P, \phi, \Delta t\right)$ describes the data $m_{k}$ is simply,

$$
-2 \ln \mathcal{L} \equiv \chi^{2}=\sum_{k}\left[\frac{m_{k}-m\left(t_{k}\right)}{\sigma_{k}}\right]^{2}
$$

A likelihood maximization, or $\chi^{2}$ minimization, determines the best-fit parameters. Phasing the measurements $m_{k}$ to a given period, we can split $\chi^{2}$ into two terms, including the points in transit and points out of transit,

$$
\chi^{2}=\sum_{i}\left(\frac{m_{i}-m_{0}+\delta}{\sigma_{i}}\right)^{2}+\sum_{j}\left(\frac{m_{j}-m_{0}}{\sigma_{j}}\right)^{2}
$$

where the first sum over index $i$ is over the points in transit, and the second over index $j$ is over the points out of transit. The quantity $\mathcal{D}$ of BLS corresponds to our $\chi^{2} / \sum_{k} \sigma_{k}^{-2}$, where the sum over index $k$ is over all points. Since $\sum_{k} \sigma_{k}^{-2}$ is a constant, this verifies the equivalence of their algorithm to a simple $\chi^{2}$-minimization. Expanding the quadratic terms in equation (2) yields,

$$
\chi^{2}=\sum_{k} \frac{m_{k}^{2}}{\sigma_{k}^{2}}-2 m_{0} \sum_{k} \frac{m_{k}}{\sigma_{k}^{2}}+m_{0}^{2} \sum_{k} \frac{1}{\sigma_{k}^{2}}+2 \delta \sum_{i} \frac{m_{i}}{\sigma_{i}^{2}}-2 \delta m_{0} \sum_{i} \frac{1}{\sigma_{i}^{2}}+\delta^{2} \sum_{i} \frac{1}{\sigma_{i}^{2}} .
$$

Given the identity $\bar{m} \equiv\left(\sum_{k} m_{k} / \sigma_{k}^{2}\right) /\left(\sum_{k} 1 / \sigma_{k}^{2}\right)=0$, it is clear that the first term is simply the $\chi^{2}$ of a constant flux fit to the data, which we denote $\chi_{0}^{2}$, and the second term is zero. Therefore, the last four terms are the improvement in $\chi^{2}$ between the constant-flux fit, and a boxcar transit fit, for a given $P, \phi, \Delta t$.

Minimizing the expression for $\chi^{2}$ with respect to $m_{0}$, we find $m_{0}=\left(\sum_{i} \sigma_{i}^{-2} / \sum_{k} \sigma_{k}^{-2}\right) \delta$. Inserting this into the expression for $\chi^{2}$, and then minimizing with respect to $\delta$, we find the parameters $\delta, m_{0}$ that simultaneously minimize $\chi^{2}$ for a given $P, \phi, \Delta t$,

$$
\begin{aligned}
\delta & =\frac{-\sum_{i} m_{i} / \sigma_{i}^{2}}{\sum_{i} \sigma_{i}^{-2}\left(1-\sum_{i} \sigma_{i}^{-2} / \sum_{k} \sigma_{k}^{-2}\right)} \\
m_{0} & =\frac{-\sum_{i} m_{i} / \sigma_{i}^{2}}{\sum_{k} \sigma_{k}^{-2}\left(1-\sum_{i} \sigma_{i}^{-2} / \sum_{k} \sigma_{k}^{-2}\right)} .
\end{aligned}
$$

We note that the solution for $\delta$ does not impose a particular sign. The best-fit boxcar model may be of a transit or anti-transit nature. The improvement in $\chi^{2}$ at these best-fit parameters is

$$
\Delta \chi^{2}=\chi^{2}-\chi_{0}^{2}=\frac{-\left(\sum_{i} m_{i} / \sigma_{i}^{2}\right)^{2}}{\sum_{i} \sigma_{i}^{-2}\left(1-\sum_{i} \sigma_{i}^{-2} / \sum_{k} \sigma_{k}^{-2}\right)} .
$$

At fixed $P, \phi, \Delta t$, and for pure Gaussian noise, $\Delta \chi^{2}$ is distributed as $\chi^{2}$ with one degree of freedom (corresponding to the one additional free parameter).

The above expression gives the $\chi^{2}$ improvement for a particular set of $P, \phi, \Delta t$. The global solution that maximizes the $\chi^{2}$ improvement, $\Delta \chi_{\max }^{2}$, requires a grid search over the entire $P, \phi, \Delta t$ parameter space feasible for transit detection. In the BLS algorithm, evaluation of $\Delta \chi^{2}$ for a given $P, \phi, \Delta t$ amounts to simple error-weighted binning, making the process extremely efficient and fast. Due to the large number of light curves with injected transits that must be searched in order to determine the detection probability accurately (see $\$ 6$ ), this study requires the excellent numerical efficiency provided by the BLS method. Comparison with BLS reveals that $\Delta \chi_{\max }^{2}$ has a close correspondence with their detection statistic SR, such that $\Delta \chi_{\max }^{2} / \sum_{k} \sigma_{k}^{-2}=-(\mathrm{SR})^{2}$. If we assume constant noise, i.e. $\sigma_{k}=$ constant $=\sigma$, and define $N_{t}$ to be the number of points in transit, then the effective signal-to-noise ratio is $\mathrm{S} / \mathrm{N}=\left(\left|\Delta \chi_{\max }^{2}\right|\right)^{1 / 2}=N_{t}^{1 / 2}(\delta / \sigma)$.

Although, at fixed $P, \phi, \Delta t$, analytic expressions can provide the significance of a given value of $\chi^{2}$ in the presence of pure Gaussian noise, in the present case folding data over many trial periods and phases hampers determination of the significance of the global $\Delta \chi_{\max }^{2}$. Thus, assessing the probability of achieving the observed outcome by chance involves taking into account the effective number of independent trials. Several papers (e.g., BLS, Jenkins et al. 2002) propose methods to address these issues. However, as we have shown, in the presence of correlated systematic errors, formulations with a Gaussian-noise basis underestimate the detection thresholds needed to avoid falsely triggering on 
the systematic errors. Generally, one must use the properties of the data themselves to set the appropriate detection threshold.

In general, a matched filter optimally detects a signal of known form in noisy data. However, the efficiency of boxcar fitting makes it highly advantageous, and as we show next nearly optimal for transit detection. This is because, for small planets and modest limb darkening, simple boxcars match well the planetary transit signal. We demonstrate this in Figure 15] where we show $\Delta \chi_{b c}^{2} \equiv \chi_{b c}^{2}-\chi_{\text {exact }}^{2}$, the difference in $\chi^{2}$ between a boxcar fit to a planetary transit across a limb-darkened star and the exact model fit, assuming uniform errors. In order to make the result independent of the error properties of the light curve, we plot this normalized to $\Delta \chi_{0}^{2} \equiv \chi_{\text {constant }}^{2}-\chi_{\text {exact }}^{2}$, the difference in $\chi^{2}$ between a constant flux fit to the light curve and the exact model fit . In other words, since $\left(\Delta \chi_{0}^{2}\right)^{1 / 2}$ is the total signal-to-noise ratio of the transit detection, the ratio $\Delta \chi_{b c}^{2} / \Delta \chi_{0}^{2}$ is (the square of) the fractional degradation of the signal-to-noise ratio that arises from fitting the approximate boxcar form to the true transit shape. We show $\Delta \chi_{b c}^{2} / \Delta \chi_{0}^{2}$ versus the impact parameter of the transit for four different values of $\rho=0.01,0.05,0.1$ and 0.2 , as well as linear limb-darkening coefficients in the range $u_{1}=0-0.4$. The fractional difference in $\Delta \chi_{0}^{2}$ is less than $\sim 5 \%$ for impact parameters $\lesssim 0.8$ and $\rho \lesssim 0.1$, and it is always $\leq 10 \%$ for the range of parameters relevant here. However, if the number of detections is a strong function of $\chi^{2}$ near the detection threshold, then even a $\sim 5 \%$ change in $\chi^{2}$ can have a significant effect on the inferred detection probability. Therefore, it is important to inject realistic transits when determining the recovery rate and detection probability, to account for the imperfect match of the boxcar filter.

\section{REFERENCES}

Aigrain, S. \& Irwin, M. 2004, MNRAS, 350, 331

Albrow, M., et al. 1998, ApJ, 509, 687

Albrow, et al. 2001, ApJ, 556, L113

Bodenheimer, P., Hubickyj, O., \& Lissauer, J. J. 2000, Icarus, 143, 2

Bond, I. A., et al. 2004, ApJ, 606, L155

Bonfils, X., et al. 2005, A\&AL, submitted (astro-ph/0509211)

Bouchy, F., Pont, F., Santos, N. C., Melo, C., Mayor, M., Queloz, D., \& Udry, S. 2004, A\&A, 421, L13

Bouchy, F., Pont, F., Melo, C., Santos, N. C., Mayor, M., Queloz, D., \& Udry, S. 2004, A\&A, 431, 1105

Bouchy, F., et al. 2005, A\&A, submitted (astro-ph/0510119)

Burke, C. J., DePoy, D. L., Gaudi, B. S., Marshall, J. L. , \& Pogge, R. W. 2003, in ASP Conf. Ser. 294, Scientific Frontiers in Research on Extrasolar Planets, ed. D. Deming \& S. Seager (San Francisco: ASP), 379

Burke, C. J., Gaudi, B. S., DePoy, D. L., Pogge, R. W., \& Pinsonneault, M. H. 2004, AJ, 127, 2382

Butler, R. P., Vogt, S. S., Marcy, G. W., Fischer, D. A., Wright, J. T., Henry, G. W., Laughlin, G., \& Lissauer, J. J. 2004, ApJ, 617,580

Carson, J. C., Eikenberry, S. S., Brandl, B. R., Wilson, J. C., \& Hayward, T. L. 2005, AJ, 130, 1212

Chabrier, G. \& Baraffe, I. 2000, ARA\&A, 38, 337

Charbonneau, D., Brown, T. M., Noyes, R. W., \& Gilliland, R. L. 2002, ApJ, 568, 377

Charbonneau, D., et al. 2005, ApJ, 626, 523

Chauvin, G., Lagrange, A. -M., Dumas, C., Zuckerman, B., Mouillet, D., Song, I., Beuzit, J. -L., \& Lowrance, P. 2005a, A\&A, 438, L25

Chauvin, G., et al. 2005b, A\&A, 438, L29

Claret, A. 2000, A\&A, 363, 1081

Crotts, A. P. S. 2001, in AAS Meet. 198, BAAS, ed. R. W. Milkey (Melville: AIP), 792

Deming, D., Brown, T. M., Charbonneau, D., Harrington, J., \& Richardson, L. J. 2005a, ApJ, 622, 1149

Deming, D., Seager, S., Richardson, L. J., \& Harrington, J. 2005b, Nature, 434, 740

Duquennoy, A. \& Mayor, M. 1991, A\&A, 248, 485

Eggenberger, A., Halbwachs, J.-L., Udry, S., \& Mayor, M. 2004, Rev. Mex. Astron. Astrofis., 21, 28

Fischer, D. A. \& Valenti, J. 2005, ApJ, 622, 1102

Gaudi, B. S. 2000, ApJ, 539, L59

Gaudi, B. S., et al. 2002, ApJ, 566, 463

Gaudi, B. S., Seager, S., \& Mallén-Ornelas, G. 2005, ApJ, 623, 472

Gilliland, R. L. \& Brown, T. M. 1988, PASP, 100, 754

Gilliland, R. L., et al. 2000, ApJ, 545, L47

Gizis, J. E., Kirkpatrick, J. D., Burgasser, A., Reid, I. N., Monet,

D. G., Liebert, J., \& Wilson, J. C. 2001, ApJ, 551, L163

Gould, A. \& Loeb, A. 1992, ApJ, 396, 104

Gould, A. \& Morgan, C. W. 2003, ApJ, 585, 1056

Guillot, T. 2005, Annu. Rev. Earth Planet. Sci., 33, 493
Halbwachs, J.-L., Mayor, M., Udry, S., \& Arenou, F. 2004, Rev. Mex. Astron. Astrofis., 21, 20

Hidas, M. G., et al. 2005, MNRAS, 360, 703

Hood, B., et al. 2005, MNRAS, 360, 791

Jenkins, J. M., Caldwell, D. A., \& Borucki, W. J. 2002, ApJ, 564, 495

Kiss, L. L. \& Bedding, T. R. 2005, MNRAS, 358, 883

Kjeldsen, H. \& Frandsen, S. 1992, PASP, 104, 413

Konacki, M., Torres, G., Jha, S., \& Sasselov, D. D. 2003, Nature, 421, 507

Konacki, M., et al. 2004, ApJ, 609, L37

Konacki, M., Torres, G., Sasselov, D. D., \& Jha, S. 2005, ApJ, 624, 372

Kovács, G., Zucker, S., \& Mazeh T. 2002, A\&A, 391, 369

Kovács, G., Bakos, G., \& Noyes, R. W. 2005, MNRAS, 356, 557

Laughlin, G., Wolf, A., Vanmunster, T., Bodenheimer, P., Fischer,

D., Marcy, G., Butler, P., \& Vogt, S. 2005, ApJ, 621, 1072

Mandel, K. \& Agol, E. 2002, ApJ, 580, L171

Mao, S. \& Paczyński, B. 1991, ApJ, 374, L37

Marcy, G. W., Butler, R. P., Fischer, D. A., Vogt, S. S., Wright, J. T., Tinney, C. G., \& Hugh, R. A. J. 2005, preprint astro-ph/0505003)

McCarthy, C. \& Zuckerman, B. 2004, AJ, 127, 2871

Mochejska, B. J., et al. 2005, AJ, 129, 2856

Narita, N., et al. 2005, PASJ, 57, 471

Neuhäuser, R., Guenther, E. W., Wuchterl, G., Mugrauer, M., Bedalov, A., \& Hauschildt, P. H. 2005, A\&A, 435, L13

Pepper, J. \& Gaudi, B. S. 2005, ApJ, 631, 581

Perryman, M. A. C. 2000, Rep. Prog. Phys., 63, 1209

Pont, F., Melo, C. H. F., Bouchy, F., Udry, S., Queloz, D., Mayor, M., \& Santos, N. C. 2005, A\&A, 433, L21

Santos, N. C., Israelian, G., \& Mayor, M. 2004, A\&A, 415, 1153

Schechter, P. L., Mateo, M., \& Saha, A. 1993, PASP, 105, 1342

Schwarzenberg-Czerny, A. 1996, ApJ, 460, L107

Seager, S. \& Mallén-Ornelas, G. 2003, ApJ, 585, 1038

Stepinski, T. F. \& Black, D. C. 2001, A\&A, 371, 250

Stetson, P. B. 1987, PASP, 99, 191

Stetson, P. B. 1996, PASP, 108, 851

Stetson, P. B., et al. 1998, ApJ, 508, 491

Tabachnik, S. \& Tremaine, S. 2002, MNRAS, 335, 151

Terquem, C., Papaloizou, J. C. B., \& Nelson, R. P. 2000, Space Sci. Rev., 92, 323

Tingley, B. \& Sackett, P. D. 2005, ApJ, 627, 1011

Udalski, A., et al. 2002, Acta Astron., 52, 1

Udalski, A., et al. 2005, ApJ, 628, L109

Udry, S., Mayor, M., \& Santos, N. C. 2003, A\&A, 407, 369

Weldrake, D. T. F., Sackett, P. D., Bridges, T. J., \& Freeman, K.

C. 2005, ApJ, 620, 1043

Yoo, J., et al. 2004, ApJ, 603, 139 
Fraction of stars with planets in NGC 1245

Table 1. MDM 2.4M Observations

\begin{tabular}{rccc}
\hline \hline Date (2001) & \#Exps & FWHM (arcsec) & Comments \\
\hline Oct. 24 & 75 & 1.2 & clear - 1st quarter moon \\
25 & 73 & 1.4 & partly cloudy \\
26 & 67 & 1.4 & partly cloudy \\
27 & 22 & 1.6 & overcast \\
28 & 96 & 1.4 & cirrus \\
29 & 86 & 1.3 & cirrus \\
31 & 32 & 1.4 & partly cloudy \\
Nov. 1 & 32 & 1.4 & clear humid - full moon \\
2 & 80 & 1.5 & clear - moon closest approach \\
6 & 44 & 1.4 & clear humid \\
7 & 92 & 1.3 & partly cloudy - 3rd quarter moon \\
8 & 57 & 1.4 & cirrus \\
10 & 81 & 1.8 & cirrus \\
11 & 99 & 1.3 & \\
& & &
\end{tabular}


Table 2. Transit Candidate data

\begin{tabular}{|c|c|c|c|c|c|c|c|c|c|c|c|c|c|c|c|c|}
\hline ID & $\mathrm{RA}(2000.0)$ & $\operatorname{Dec}(2000.0)$ & $V(\mathrm{mag})$ & $B-V(\mathrm{mag})$ & $V-I$ (mag) & $\chi_{\text {mem }}^{2}$ & $P(\mathrm{~d})$ & $\Delta f(\mathrm{mag})$ & $\tau(\mathrm{h})$ & $\phi$ & $\Delta \chi^{2} / \Delta \chi_{-}^{2}$ & $\Delta \chi^{2}$ & $f$ & $M\left(\mathrm{M}_{\odot}\right)$ & $\log \left(\left(\mathrm{R} / \mathrm{R}_{\odot}\right)\right.$ & $\operatorname{Teff}(\mathrm{K})$ \\
\hline 30207 & $3: 15: 40.0$ & $+47: 21: 18$ & 18.1 & 1.17 & 1.31 & 0.137 & 4.614 & 0.030 & 1.66 & 0.72 & 5.65 & 584 & 0.57 & & & $\ldots$ \\
\hline 20513 & $3: 15: 04.6$ & $\begin{array}{l}+47: 15: 09 \\
\end{array}$ & 18.6 & 1.10 & 1.27 & 0.028 & 1.637 & 0.018 & 4.71 & 0.95 & 3.85 & 840 & 0.49 & 0.91 & -0.095 & 5400 \\
\hline 20065 & 3:15:03.8 & $\begin{array}{l}+47: 14: 33 \\
\end{array}$ & 16.1 & 1.02 & 1.25 & 0.418 & 3.026 & 0.115 & 4.18 & 0.24 & 3.82 & 88086 & 0.59 & & $\ldots$ & 急 \\
\hline 20398 & $3: 14: 49.5$ & $+47: 16: 03$ & 18.4 & 1.29 & 2.00 & 0.863 & 0.349 & 0.145 & 0.90 & 0.72 & 3.76 & 66056 & 0.29 & $\ldots$ & $\ldots$ & $\ldots$ \\
\hline 20274 & $3: 14: 35.9$ & $+47: 19: 29$ & 19.3 & 1.67 & 3.37 & 4.390 & 0.302 & 0.050 & 0.98 & 0.48 & 3.23 & 14063 & 0.21 & $\ldots$ & $\ldots$ & $\ldots$ \\
\hline 70718 & $3: 13: 56.2$ & $+47: 06: 59$ & 21.1 & 1.28 & 1.79 & 0.017 & 0.640 & 0.032 & 3.07 & 0.16 & 2.86 & 312 & 0.22 & 0.63 & -0.253 & 4300 \\
\hline
\end{tabular}

